\title{
Definition and Basic Properties of the Deva Meta-Calculus
}

\author{
Matthias Weber ${ }^{1}$ \\ Technische Universität Berlin, Berlin, Germany.
}

Keywords: Formalization of development methods; Logical frameworks

\begin{abstract}
This article presents the definition and some basic properties of the Deva meta-calculus, a generic logical framework whose design was driven by the needs arising from the instantiation to software development methods. As a result, Deva contains structures that do not occur in comparable logical frameworks. There now exist a number of case studies about the formalization of software development methods in Deva. In this article, a structured definition of Deva is presented and basic parts of its language theory, viz Church-Rosser, closure, and strong normalization, are summarized.
\end{abstract}

\section{Introduction}

The current situation with respect to the application of formal software development methods is characterized by a partial utilization of the benefits offered by the formal approach to software development: more and more systems are successfully specified in formal notations, however formal developments down to efficient implementations and inclusive of all necessary proofs are still very rare.

The formal specification of software systems has been relatively successful because it helps yield precise knowledge and understanding of the problem to be solved: information which will effect significant benefits in the subsequent development phase. The formal development of software systems, in the above sense, has been much less successful because with growing size of examples, the transitions and proofs quickly become too complex to be effectively understood

\footnotetext{
${ }^{1}$ Major parts of the work reported here were carried out while the author was under contract at the German National Research Centre (GMD) in Karlsruhe and at the University of Karlsruhe Correspondence and offprint requests to: Matthias Weber, Technische Universität Berlin, FB20, FR 5-6, 1000 Berlin 10, Germany. email: we@cs.tu-berlin.de
} 
and managed, regardless of the degree of automatic support. The enormous amount of necessary proofs, which are mostly of technical nature, is, in the author's view, not inherent to the software development process as such, but is due to a lack of well designed and generally accepted methods and notation, a lack of a library of generally accepted standard theories for software development, and a lack of tools which support all this.

On the other hand, theoretical research has produced remarkable progress towards the development and investigation of powerful generic proof frameworks, or meta-calculi, based on typed $\lambda$-calculus, that offer a natural and universal formal development machinery and can be instantiated by various logical and mathematical theories ([Bru80], [CH85], [HHP86]). Most of these frameworks have been designed and used within a theoretical environment, to study issues of formalization and proof.

This article reports on the definition and theoretical investigation of a generic development framework designed to express completely formal developments. This framework, named Deva meta-calculus, can be seen as a contribution towards linking work performed on proof frameworks with work on formal development methods. The connection consists of viewing developments as proofs and development methods as theories. As a result, development methods become completely formalized objects. The purpose of Deva is to experiment with complete formalizations of currently known methods and with the resulting method support systems. This experimentation can be a quite rewarding process, e.g. by pointing out informal parts both in the method itself and in its use. Similarly, it is interesting to experiment with libraries of basic theories for software developments based on the method.

While our investigations about requirements and design of a development calculus dates back to the work of Michel Sintzoff [Sin80], the Deva metacalculus presented in this article was developed mainly between 1987 and 1989 in the context of the ESPRIT-project ToolUse [SWGC89]. The objective of the ToolUse project was to study a broad spectrum of development methodologies (e.g. Jacksons System Design, the Vienna Development Method, or BurstallDarlington's fold/unfold method for program transformation) and to design a method-driven support environment. Deva was then intended to serve as a notational framework to help clarify the understanding of such methods. The language was developed by a collaborative effort of three different subgroups of the project: a group headed by Michel Sintzoff at the University of Louvain (UCL), a group headed by Renée Jacquart at the French research centre for technology (CERT) in Toulouse, and a group headed by Stefan Jähnichen at the German research centre for computer science (GMD) in Karlsruhe. There now exist a number of case studies about the formalization of development methods in Deva ([BS91], [Gab91], [Laf90], [Web90], [Web91b]). Recently, an introduction to Deva and two extensive case studies have been compiled into a book [WSL93]. The language theory for a kernel calculus of Deva was developed by Philippe de Groote and is described in [Gro90]. This article deals with the full calculus as investigated in [Web91a].

The essential technical requirements driving the design of Deva have been the following:

- It must be possible to instantiate Deva to an interesting range of methods. Thus, it should offer a generic logical system since many methods are based on completely different logical systems. 
- Deva should offer an integrated linguistic framework to express specifications, programs, proofs, developments, and tactics as well as properties of these objects.

- In addition to basic logical concepts, there must be structural concepts to help meet practical needs during the construction and management of (usually large) developments.

- Deva should be a consistent framework in the sense that the only possible source of inconsistencies in Deva descriptions should be the formalized methods and not the Deva language itself.

The first requirement, i.e. logical genericity and default-freeness, has been met by designing Deva as a logical framework, reusing research results about the AUTOMATH family of languages [Ned80]. Since logical frameworks offer a (at least partial) synthesis of formulas, proofs, and theories in a common notation, the second requirement, namely building an integrated linguistic framework, was also partially met. Tactics, however, are not part of logical frameworks but usually of their support systems. In order to achieve an integration of tactical proof aspects, an implicit level of notation has been added to Deva. At the implicit level, developments need not be described in full detail. For example, rule parameters can be omitted on application of a rule. Similarly, the choice between several rules and the number of times a rule is applied may be left unspecified. This approach allows smooth integration of tactical proof aspects into the notation.

The third requirement was met by introducing additional proof constructs and a simple notion of theory: the basic operations of typed $\lambda$-abstraction and application, present in all logical frameworks, were augmented by static type assertions, products (with projection and update), sums (with injection and case distinction), and logical cuts. Operations to structure theories include a basic mechanism to present theories in a modular fashion, renaming of variables, and abstraction and instantiation of theories. We will show that these concepts provide a number of useful operations which we think are indispensable for the formalization of development methods. The price to be paid for these concepts is that they make the formal definition of the Deva language more complex than that of ordinary proof frameworks.

The last requirement is met by defining a notion of normal form of Deva descriptions and exhibiting a procedure to obtain an equivalent normal form from every Deva description. It is proven that every correctly-typed description has a unique equivalent normal form which can be effectively computed in finite time. The structure of normal forms is relatively simple and makes it easy to see that no new inconsistencies are introduced into theories by the use of Deva.

The article is structured as follows:

- Two small examples are presented and explained by appealing to the reader's intuition. The main goal is to provide a flavor of what the notation can express and how it can be used. The examples will later be explained in depth as an illustration of the formal definition of Deva. This section is meant as an appetizer rather than a tutorial.

- The second section defines the explicit part of Deva, consisting essentially of the explicit operations and the definition of the semantic relations reduction, typing, and explicit (type) validity.

- The third section defines the implicit part of Deva, a syntactic extension of the explicit part which introduces several new implicit operations and their 
validity. Validity of the implicit operations is justified by their explanation in terms of valid explicit operations.

- The fourth section outlines the proof of some fundamental properties of Deva and two applications of these properties.

\section{Two Examples}

The first example (Fig. 1) presents a proof of Syllogism within a formalization of minimal logic. First, minimal logic is formalized in a natural deduction style: Propositions (prop) are the only syntactic class and implication $(\Rightarrow)$ is the only constructor for propositions. The theorems of minimal logic are characterized by two inference rules for the introduction and elimination of implications. The introduction rule allows to infer $(p \Rightarrow q)$ from a proof of $q$ under the assumption $p$, i.e. the assumption $p$ is resolved. The elimination rule does just the reverse.

The formalization of minimal logic is then imported and syllogism (i.e. transitivity of implication) is proven. The proof runs as follows: assume, for arbitrary propositions $a, b$, and $c$, that $a \Rightarrow b$ and $b \Rightarrow c$ holds. Using the elimination rule on each of these assumptions, one infers that $b$ holds under the assumption of $a$ and $c$ holds under the assumption of $b$. By resolving the intermediate formula $b$, one infers that $c$ holds under the assumption of $a$. The proof of $a \Rightarrow c$ is then obtained by applying the introduction rule.

An almost trivial simplification tactic for boolean expressions is described and illustrated in Fig. 2. Two simplification rules are considered:

$(a \wedge t r u e)$ is replaced by $a \quad$ and $\quad(a \vee f a l s e)$ is replaced by $a$.

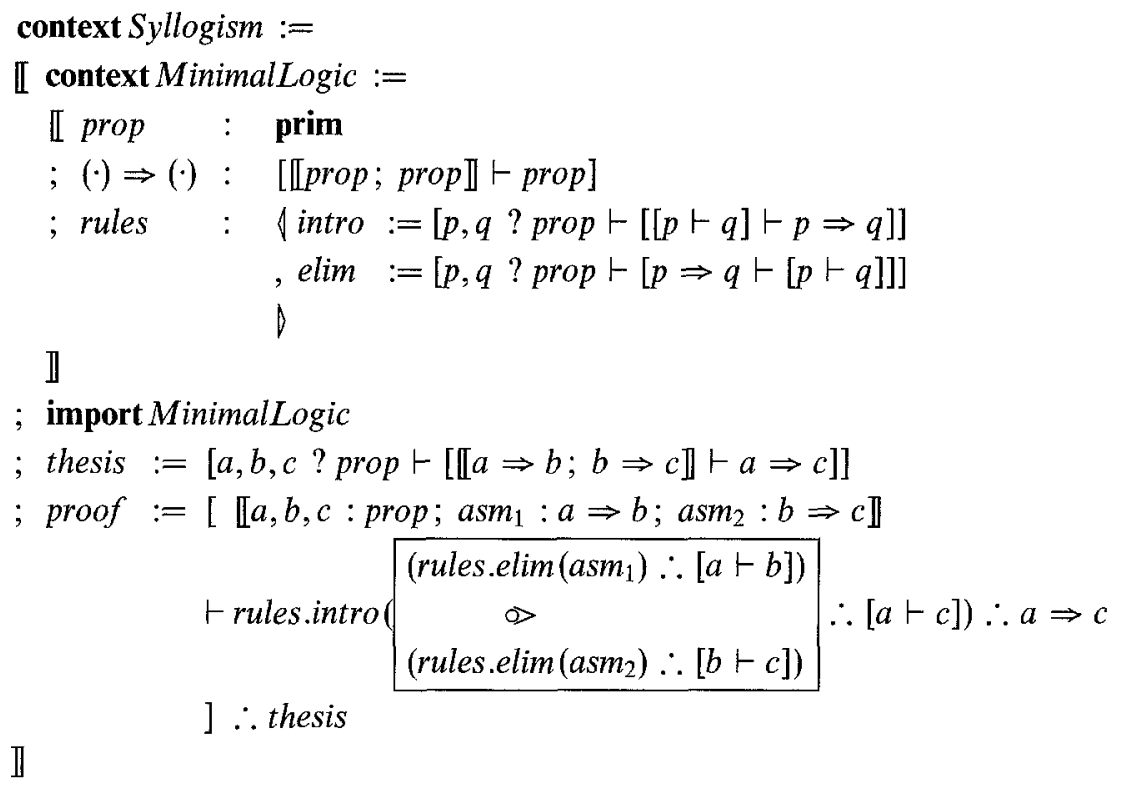

Fig. 1. Proof of Syllogism in minimal logic. 


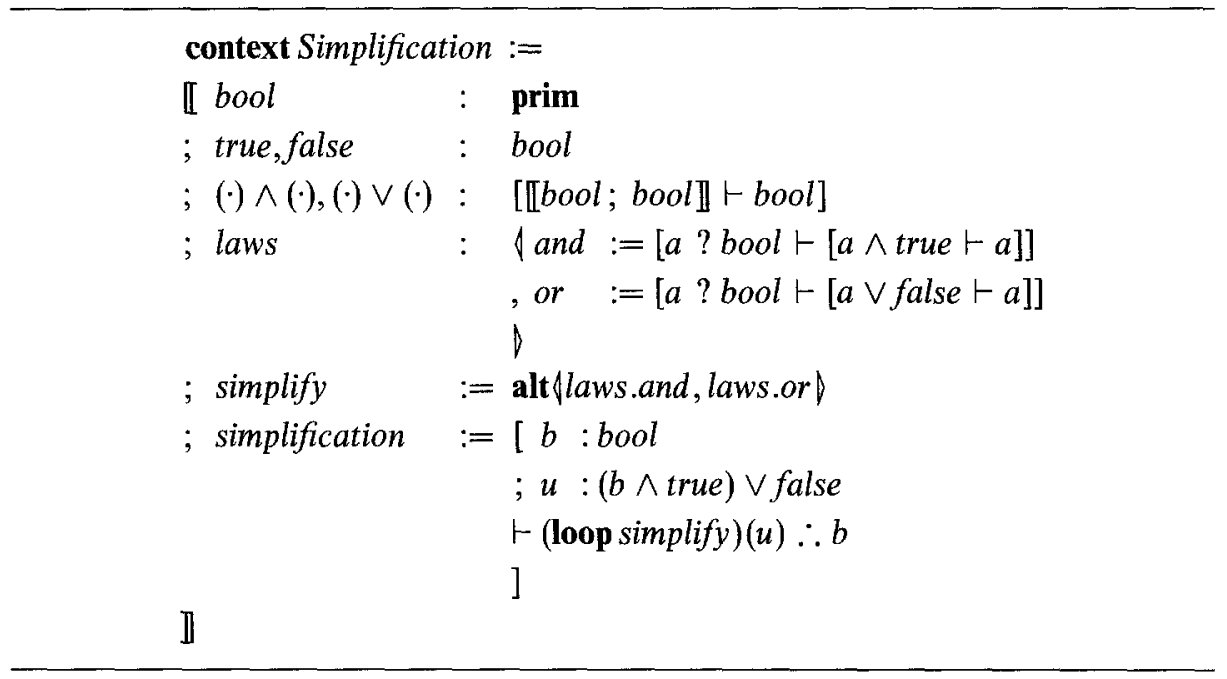

Fig. 2. Simplification of boolean expressions.

A simplification tactic (simplify) is defined which may alternatively choose between one of these rules. Its use is illustrated on a simple example in which a proof $u$ of $(b \wedge$ true $) \vee$ false is transformed into a proof of $b$.

\section{Definition of the Explicit Part of Deva}

Quite obviously, some parts of the examples of section 1 were not presented in a completely explicit way. For example, in the syllogism proof some parameters of the introduction and elimination rules were not instantiated. Similarly, the second example provided only rather rough information about the simplification itself. For these reasons, the examples have been expressed using the implicit part of Deva.

This section presents the sublanguage of Deva that covers completely explicit developments. After introducing the syntax and informally sketching the intended meaning of the explicit part, the examples are reexpressed in a completely explicit setting.

\subsection{Formation}

Let $\mathscr{V}_{t}$ denote the (enumerable) set of text identifiers, $\mathscr{V}_{c}$ the enumerable set of context identifiers, and $\mathbb{N}$ the positive natural numbers (the three sets are assumed to be disjoint), then the syntax of the explicit part of Deva is made up of two syntactic classes, texts denoted by $\mathscr{T}$ and contexts denoted by $\mathscr{C}$. The syntax of the texts and contexts of the explicit part is specified by the following grammar:

$$
\begin{aligned}
\mathscr{T}:= & \text { prim } \\
& \mid \mathscr{V} t \\
& \mid \mathscr{C} \vdash \mathscr{T}] \\
& \mid \mathscr{T}(\mathbb{N}:=\mathscr{T})
\end{aligned}
$$

primitive text

text identifier

abstraction

application 


$$
\begin{array}{ll}
\mid \mathscr{T} \therefore \mathscr{T} & \text { judgement } \\
\left|\mathscr{V}_{t}:=\mathscr{T}, \ldots, \mathscr{V}_{t}:=\mathscr{T}\right\rangle & \text { named product } \\
\mid \mathscr{T} . \mathbb{N} & \text { projection } \\
\mid \mathscr{T} \text { where } \mathbb{N}:=\mathscr{T} & \text { product modification } \\
\mid\left\langle\mathscr{V}_{t}:={ }^{k} \mathscr{T}|\ldots| \mathscr{V}_{t}:=\mathscr{T}|\ldots| \mathscr{V}_{t}:={ }^{k} \mathscr{T}\right| & \text { named sum, } k \geq 0 \\
\mid \text { case } \mathscr{T} \text { of } \mathscr{T} & \text { case distinction } \\
\mid \mathscr{T} \text { upto } \mathbb{N} \gg \mathscr{T} \text { at } \mathbb{N} & \text { cut }
\end{array}
$$

The productions have been labeled for further reference. Named products are meant to contain at least one component $\mathscr{V}_{t}:=\mathscr{T}$ and named sums are meant to contain, for a fixed $k$, at least one component $\mathscr{V}_{t}:={ }^{k} \mathscr{T}({ }^{k}$ stands for $\underbrace{\ldots \hat{j}}_{k})$ and exactly one component $\mathscr{V}_{t}:=\mathscr{T} \cdot{ }^{0} t$ is the same as $t$. Note that the above grammar has an infinite set of productions.

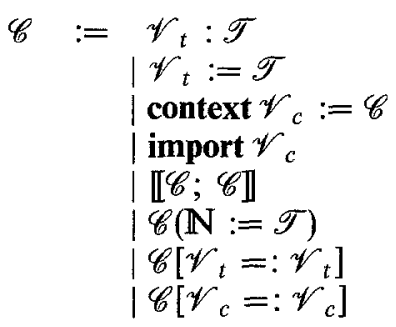

declaration
definition
context definition
context inclusion
context join
context application
renaming

In the following discussions, let $n$ and $m$ range over $\mathbb{N}, x$ and $y$ over $\mathscr{V}_{t}, p$ and $q$ over $\mathscr{F}_{c}, t$ over $\mathscr{T}$, and $c$ over $\mathscr{C}$. Also, the indexed variants of these variables shall range over the same sets, e.g. $x_{1}$ is supposed to range over $\mathscr{V}_{t}$. Further, define the set of expressions $\mathscr{E}$ to be $\mathscr{T} \cup \mathscr{C}$ and define the set of identifiers $\mathscr{V}_{e}$ to be $\mathscr{V}_{t} \cup \mathscr{V}_{c}$. Let $e$ range over $\mathscr{E}$ and $X, Y$ and $Z$ range over $\mathscr{V}_{e}$.

\subsection{Intended Meaning of the Constructs}

In general, contexts are the means of the Deva meta-calculus to construct calculi, i.e. formalized theories. Among the theories that have been formalized with contexts are: classical and non-classical logics, basic mathematical theories, algebraic data types, and precise methods for software development such as transformation and refinement methods.

A declaration $x: t$ introduces the identifier $x$ of type $t$. As will be seen below, several operations may instantiate this identifier to a text of type $t$. A definition $x:=t$ introduces an identifier $x$ as an abbreviation for the text $t$. A context definition context $p:=c$ introduces an identifier $p$ as an abbreviation for the context $c$. The context inclusion import $p$ of an applied context identifier $p$ stands for the context $c$ given in the corresponding context definition context $p:=c$. In a context join $\llbracket c_{1} ; c_{2} \rrbracket$, the declarations and definitions in $c_{1}$ are made visible in $c_{2}$. Thus text and context identifiers introduced in $c_{1}$ may be used in the declarations and definitions of $c_{2}$ (but not conversely). A context application $c(n:=t)$ instantiates the $n$-th declared identifier in $c$ to the text $t$, provided the declared type and the actual type conform. A renaming $c[x=: y]$ renames the text identifier $x$ to $y$ in $c$. A renaming $c[p=: q]$ renames the context identifier $p$ to $q$ in $c$. 
The laws defined by contexts may be applied to construct calculations, i.e. derivations, proofs, or developments. Such calculations are called texts.

The primitive text prim is the starting point for the hierarchical type construction process in Deva. Any applied occurrence $x$ of a text identifier must have a corresponding declaration or definition. In an abstraction $[c \vdash t]$, the context $c$ can be seen as a set of parameter declarations or a set of assumptions, whereas the text $t$ can be seen as a function body or a conclusion. Thus, the abstraction $[c \vdash t]$ plays the role of a function or an inference rule. An application $t_{1}\left(n:=t_{2}\right)$ instantiates the (multi-ary) function $t_{1}$ at its $n$-th domain, provided the declared type and the actual type conform. A judgement $t_{1} \therefore t_{2}$ is a text $t_{1}$ together with the assertion that its type is $t_{2}$. A named product introduces a finite list of named texts. Projection allows specializing of a product to a particular instance. Product modification modifies a component of a product. In addition to the named product, the named sum is another construct to collect a finite list of texts. Sums come in several variants: we will call a sum like the one in the grammar above an $r$-injection. In the special case where $r$ is zero, we also use the term plain sum. One can think of a plain sum $\left|x_{1}:=t_{1}\right| x_{2}:=t_{2} \mid$ to model the disjoint union, or variant record, of all texts whose type is equal to $t_{1}$ and all texts whose type is equal to $t_{2}$. If the type of, say, $t_{1}^{\prime}$ is convertible to $t_{1}$ the 1 -injection $\left.\left|x_{1}:=t_{1}^{\prime}\right| x_{2}:={ }^{\wedge} t_{2}\right\rangle$ then stands for the tagged element $t_{1}^{\prime}$ of $\left\langle x_{1}:=t_{1} \mid x_{2}:=t_{2}\right\rangle$. Typing in Deva will essentially be defined as a homomorphic mapping from texts to texts (cf. 2.9) ! As a consequence " $r$-th types" of texts can be constructed by repeatedly applying the type mapping $r$ times to texts. This allows introducing generalized $r$-injections, behaving as 1 -injections, except that the $r$-th type of the unrealized variants is specified. In general, a sum has properties weaker than those of the product, i.e. projection is not allowed. Instead, by case distinction case $t_{1}$ of $t_{2}$ one may select from a number of alternative functions in $t_{2}$, depending on the type of the argument $t_{1}$. Sums control the static description of these alternatives as well as the selection process. Whereas application is a point level operation between, i.e. a text acting as a function applied to a text acting as an argument, cuts introduce a way to compose two texts, both acting as functions. For example, the text

$$
[x: t \vdash f(1:=x)] \text { upto } 1 ه\left[y: t^{\prime} \vdash g(1:=y)\right] \text { at } 1
$$

is intuitively the same as $[x: t \vdash g(1:=f(1:=x))]$. The general case simply allows specification of the concrete argument positions at which the composition shall take place. The name "cut" has been chosen, because the behavior is very akin to that of the cut-rule in natural deduction. For example the reduction of a cut (cf. 2.7) resembles cut elimination.

\subsection{Fully Explicit Presentation of the Examples}

Figs. 3 and 4 present the examples from section 1 in full detail using the constructs from the explicit part of Deva. The reader should compare the explicit versions with their corresponding implicit versions. A precise technical relation between implicit and explicit expressions will be defined in section 3. Some syntactic sugar has been used to enhance readability. In Fig. 3 , the declaration $(\cdot) \Rightarrow(\cdot)$ allows to apply $\Rightarrow$ in infix notation, i.e. $p \Rightarrow q$ is equivalent syntax for $(\Rightarrow(1:=p))(1:=q)$. This double application describes the instantiation of $\Rightarrow$ to $p$ at its first domain and of $(\Rightarrow(1:=p))$ to $q$ at its first domain (which amounts to instantiating $\Rightarrow$ 


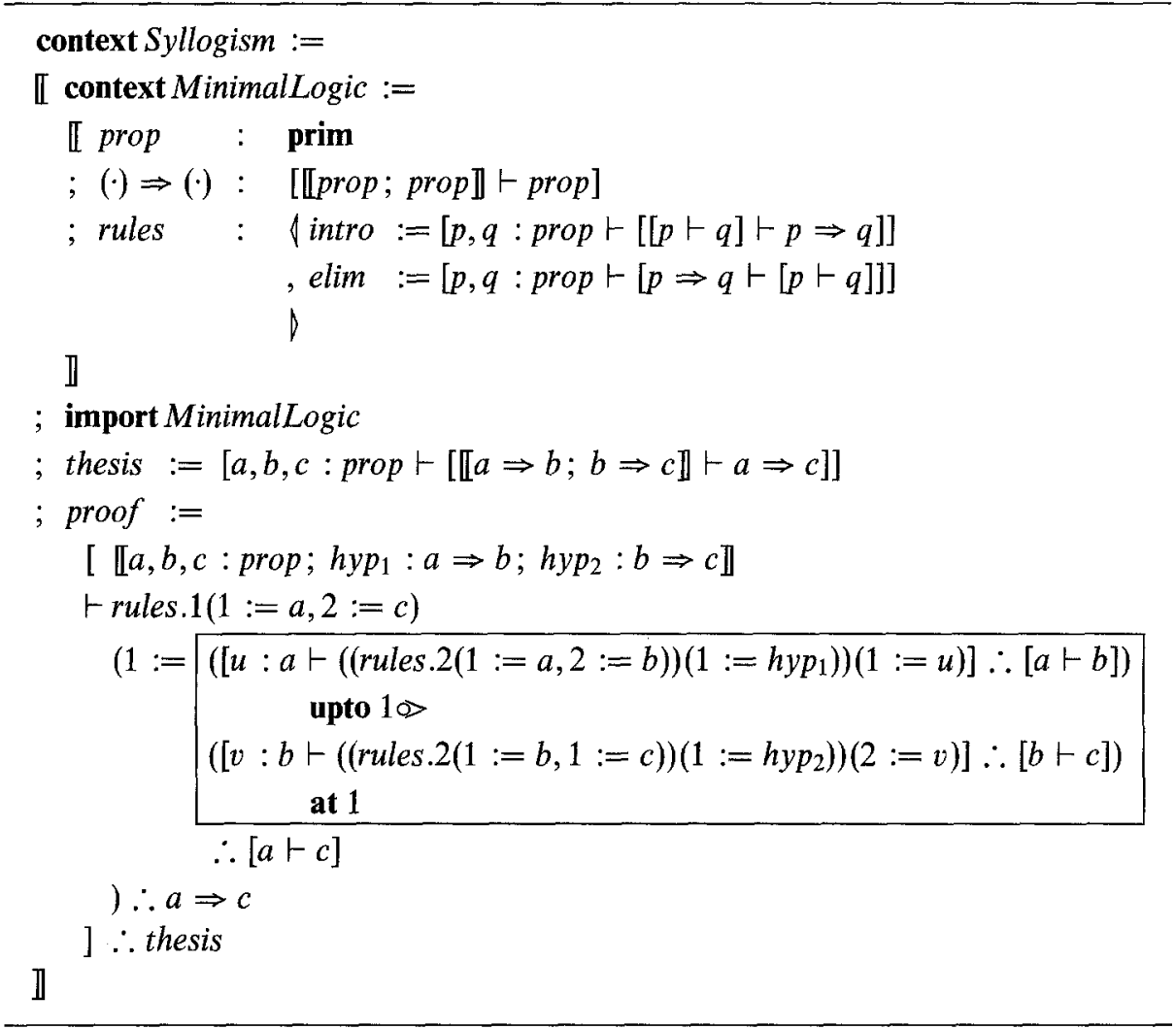

Fig. 3. Explicit version of Fig. 1.

to $q$ at its second domain). Furthermore, the following equivalent syntax is used:

$$
\begin{aligned}
\llbracket c_{1} ; c_{2} ; \cdots ; c_{n-1} ; c_{n} \rrbracket & \simeq \llbracket c_{1} ; \llbracket c_{2} ; \cdots ; \llbracket c_{n-1} ; c_{n} \rrbracket \cdots \rrbracket \rrbracket \\
{\left[t_{1} \vdash t_{2}\right] } & \simeq\left[x: t_{1} \vdash t_{2}\right] x \text { new } \\
{\left[\llbracket t_{1} ; t_{2} \rrbracket \vdash t\right] } & \simeq\left[\llbracket x: t_{1} ; y: t_{2} \rrbracket \vdash t\right] \quad x \text { and } y \text { new } \\
x, y: t & \simeq \llbracket x: t ; y: t \rrbracket \\
t\left(1:=t_{1}, 2:=t_{2}\right) & \simeq\left(t\left(1:=t_{1}\right)\right)\left(1:=t_{2}\right) \\
{\left[t_{1}, t_{2}\right] } & \simeq\left[x:=t_{1}, y:=t_{2}\right] \quad x \text { and } y \text { new } \\
{\left[t_{1} \mid t_{1}\right] } & \simeq\left[x:=t_{1} \mid y:=t_{2}\right] \quad x \text { and } y \text { new }
\end{aligned}
$$

The last convention for sums is used similarly for injections. Note that the explicit version of minimal logic differs from the implicit one only in the use of colons within declarations (declarations with question-mark will be defined in section 3).

The explicit version of the Syllogism proof contains several new applications and abstractions. Nevertheless the overall structure of the proof has been preserved. In contrast, Fig. 4 is a rather significant expansion of the implicit version of the simplification example of Fig. 2. The following sections will illustrate the semantic notions of the explicit part by using various excerpts from these examples. 


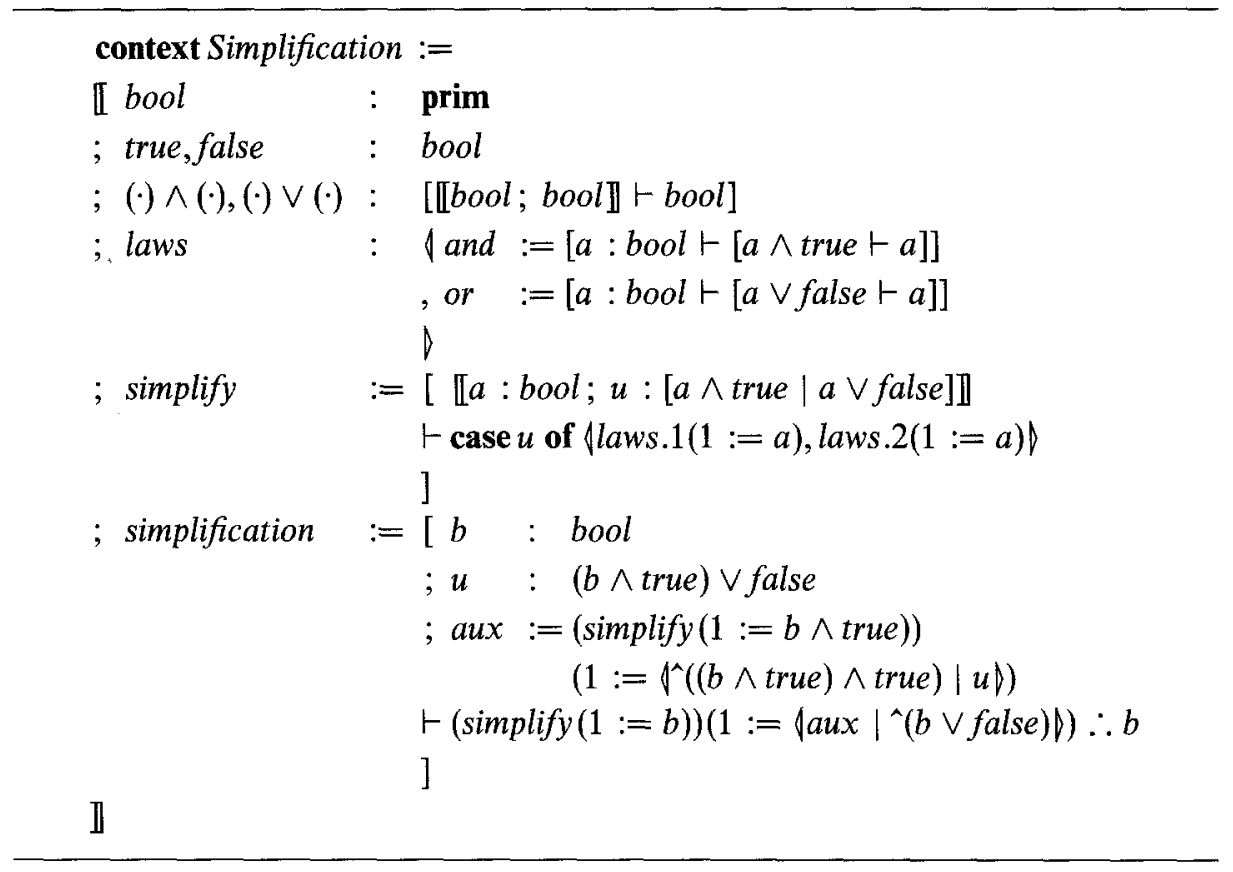

Fig. 4. Explicit version of Fig. 2.

\subsection{Environments}

Declarations, definitions, and context definitions are called bindings. In its general form, a context is a structured collection of bindings. For the definitions of the semantic assertions, at some places it would be technically very complex to work with contexts, so that the structure of a context is partially evaluated into a linear list of bindings called environments. Let $\mathscr{B} \mathscr{N} \mathscr{D}$ denote the subset of bindings of $\mathscr{C}$ and let $\mathscr{E} \mathscr{N} \mathscr{V}$ denote the data structure determined by the following operations:

$$
\begin{aligned}
& \langle\rangle: \quad \rightarrow \mathscr{E} \mathscr{N} \mathscr{V}, \quad \text { empty environment } \\
& \langle(\cdot)\rangle: \mathscr{B} \mathscr{N} \mathscr{D} \quad \rightarrow \mathscr{E} \mathscr{N} \mathscr{V}, \quad \text { singleton environment } \\
& (\cdot) \oplus(\cdot) \quad: \quad \mathscr{E} \mathscr{N} \mathscr{V} \times \mathscr{E} \mathscr{N} \mathscr{V} \rightarrow \mathscr{E} \mathscr{N} \mathscr{V}, \quad \text { environment concatenation }
\end{aligned}
$$

where $\oplus$ is associative and has the identity \langle\rangle . From now on, $E$ is assumed to range over environments.

The visibility rules of Deva are based on a lookup function $E \uparrow X$ which identifies the binding determined by an identifier $X$ within an environment $E$. If $X \odot e$ denotes a binding, i.e. either $X: e(\mathrm{C} 1)$, or $X:=e(\mathrm{C} 2)$, or context $X:=e$ (C3), then the following two equations completely determine this function:

$$
\begin{aligned}
\langle\rangle \uparrow Y & =\text { undefined } \\
(E \oplus\langle X \odot e\rangle) \uparrow Y & =\left\{\begin{array}{cl}
X \odot e & \text { if } X=Y \\
E \uparrow Y & \text { otherwise. }
\end{array}\right.
\end{aligned}
$$

Now, the operation symbol $\oplus$ is overloaded to also account for the operation $E \oplus c$ of "pushing a context $c$ onto an environment $E$ " by partially evaluating the context operations in $c$. The context operations are the binding of an identifier 
to an expression ( $\mathrm{CI}, \mathrm{C} 2, \mathrm{C} 3)$, the use of a context variable $p(\mathrm{C} 4)$, the join of two contexts (C5), the application of a context to a text (C6), and the renaming of an identifier in a context $(\mathrm{C} 7, \mathrm{C} 8)$. The following rules completely determine the operation $E \oplus c$ :

$$
\begin{gathered}
\overline{E \oplus(X \odot e)=E \oplus\langle X \odot e\rangle} \\
\frac{E \uparrow p=(\text { context } p:=c)}{E \oplus \text { import } p=E \oplus c} \\
\overline{E \oplus \llbracket c_{1} ; c_{2} \rrbracket=\left(E \oplus c_{1}\right) \oplus c_{2}} \\
\frac{E \oplus c=E \oplus\left\langle x: t_{1}\right\rangle \oplus E_{1}}{E \oplus c(1:=t)=E \oplus\langle x:=t\rangle \oplus E_{1}} \\
\frac{E \oplus c=E \oplus\left\langle x: t_{1}\right\rangle \oplus c_{1}}{E \oplus c(n+1:=t)=E \oplus\left\langle x: t_{1}\right\rangle \oplus c_{1}(n:=t)} \\
\frac{E \oplus c=E \oplus\left\langle x:=t_{1}\right\rangle \oplus c_{1}}{E \oplus c(n:=t)=E \oplus\left\langle x:=t_{1}\right\rangle \oplus c_{1}(n:=t)} \\
\frac{E \oplus c=E \oplus\left\langle\text { context } p:=c_{2}\right\rangle \oplus c_{1}}{E \oplus c(n:=t)=E \oplus\left\langle\text { context } p:=c_{2}\right\rangle \oplus c_{1}(n:=t)} \\
E \oplus c=E \oplus\langle Y \odot e\rangle \oplus E_{1} \\
\hline E \oplus c[Y=: Z]=E \oplus\langle Z \odot e\rangle \oplus E_{1} \\
E \oplus c=E \oplus\langle X \oplus e\rangle \oplus c_{1} \quad X \neq Y \\
\hline E \oplus c[Y=: Z]=E \oplus\langle X \oplus e\rangle \oplus c_{1}[Y=: Z]
\end{gathered}
$$

Here, it is assumed that expressions of the form $E_{1} \oplus E_{2} \oplus c$ associate to the left, i.e. they stand for $\left(E_{1} \oplus E_{2}\right) \oplus c$. As can be seen from the above rules, an application $c(n:=t)$ amounts to the replacement of the $n$-th declaration $x: t_{1}$ in $c$ by the definition $x:=t$ and a renaming $c[Y=: Z]$ amounts to the replacement of the first binding $Y \odot e$ in $c$ by the binding $Z \odot e$.

Illustration. The importation of minimal logic in Fig. 3 is easily computed as follows:

$$
=\begin{aligned}
& \langle\text { context MinimalLogic }:=\cdots\rangle \oplus \text { import MinimalLogic } \\
& \{\mathrm{P} 2\} \\
& \langle\text { context MinimalLogic }:=\cdots\rangle \oplus \llbracket \text { prop : prim; } \cdots \rrbracket \\
& \{\mathrm{P} 3, \mathrm{PI}\} \\
& \langle\text { context MinimalLogic }:=\cdots\rangle \oplus\langle\text { prop : prim }\rangle \oplus \mathbb{I} \cdots \mathbb{}
\end{aligned}
$$

Remark. Note that the rule P4 does not require that the argument $t$ is "typecompatible" with the formal parameter $t_{1}$. Indeed, at the current point of definition no typing constraints are considered. However, typing constraints will be systematically defined and imposed on expressions further below (cf. 2.11).

Remark. If one carefully looks at the above rules, one will notice a naming problem caused by the rule P2: straightforward implementation of this rule could lead to name clashes, if, for instance, the imported context $c$ depends on some identifier which is redefined in $E$ after the definition of $p$. For the moment, the problems involved with naming are deferred (cf. 2.8) and it is appealed to the 
intuition of the reader that in any given text or context the names of identifiers can be chosen in such a way that no name clashes occur.

In order to ensure that the renaming operation is always defined on contexts, a predicate which expresses that a context $c$ 'binds' an identifier $X$ relative to an environment $E$, denoted by $\operatorname{binds}_{E}(c, X)$, is introduced. It is determined by the following rule:

$$
\frac{E \oplus c=E \oplus E_{1} \oplus(X \odot e) \oplus E_{2}}{\operatorname{binds}_{E}(c, X)}
$$

\subsection{Homomorphic Extension}

The subsequent definitions will introduce a number of semantic relations on expressions. Many of the rules defining these relations are constructed according to a general pattern derived from the structure of the language: An expression is in the relation if all its subexpressions are and if some additional relation between its subexpressions holds. It should be sufficient to state only this additional relation for each construct of the language since the rest of the rule is determined by the structure of the construct. As such, this is not quite exact since we have to distinguish two different types of constructs: sequential constructs such as abstraction, and parallel constructs such as application. These two types induce structurally different rules because subexpressions of sequential constructs may depend on each other whereas subexpressions of parallel constructs do not.

To express this formally, let $\mathscr{S} \mathscr{E} \mathscr{Q}$ denote the set of sequential phrases specified by the grammar

$$
\mathscr{S} \mathscr{E} \mathscr{Q}:=\left[\mathscr{C}_{1} \vdash \mathscr{T}_{2}\right] \mid \llbracket \mathscr{C}_{1} ; \mathscr{C}_{2} \rrbracket,
$$

and let $\mathscr{P} \mathscr{A} \mathscr{R}_{\text {ex }}$ denote the set of parallel phrases of the explicit part specified by the grammar

$$
\begin{aligned}
\mathscr{P} \mathscr{A} \mathscr{R}_{e x}:= & \operatorname{prim}\left|\mathscr{V}_{t}\right| \mathscr{T}_{1}\left(\mathbb{N}:=\mathscr{T}_{2}\right)\left|\mathscr{T}_{1} \therefore \mathscr{T}_{2}\right| \\
& \left|\mathscr{V}_{t}:=\mathscr{T}_{1}, \ldots, \mathscr{V}_{t}:=\mathscr{T}_{k}\right|\left|\mathscr{T}_{1}, \mathbb{N}\right| \mathscr{T}_{1} \text { where } \mathbb{N}:=\mathscr{T}_{2} \\
& \left|\mathscr{V}_{t}:=\mathscr{T}_{1}\right| \cdots\left|\mathscr{V}_{t}:=\mathscr{T}_{k}\right| \mid \text { case } \mathscr{T}_{1} \text { of } \mathscr{T}_{2} \mid \\
& \mathscr{T}_{1} \text { upto } \mathbb{N} \gg \mathscr{T}_{2} \text { at } \mathbb{N} \mid \\
& \mathscr{V}_{t}: \mathscr{T}_{1}\left|\mathscr{V}_{t}:=\mathscr{T}_{1}\right| \text { context } \mathscr{V}_{c}:=\mathscr{C}_{1} \mid \text { import } \mathscr{V}_{c} \mid \\
& \mathscr{C}_{1}\left(\mathbb{N}:=\mathscr{T}_{2}\right)\left|\mathscr{C}_{1}\left[\mathscr{V}_{t}=: \mathscr{V}_{t}\right]\right| \mathscr{C}_{1}\left[\mathscr{V}_{c}=: \mathscr{V}_{c}\right] .
\end{aligned}
$$

Here, the indices given to the sets $\mathscr{T}$ and $\mathscr{C}$ specify the argument positions of the subexpressions of the particular construct. Letting $e$ denote arbitrary expressions i.e. either a text or a context, this allows an element of $\mathscr{S} \mathscr{E} \mathscr{Q}$ to be specified by $c \Theta e$, and an element of $\mathscr{P} \mathscr{A} \mathscr{R}_{e x}$ by $\circledast_{e x}\left(e_{1}, \ldots, e_{k}\right)$ for some $k \geq 0$. Thus, $\circledast_{e x}\left(t_{1}, t_{2}\right)$ may stand e.g. for $t_{1}\left(x:=t_{2}\right), \circledast_{e x}(t)$ may stand e.g. for $x: t$, and $\circledast_{e x} 0$ may stand e.g. for prim or any text identifier $x$.

Given predicates $P_{\circledast_{e x}}\left(E, e_{1}, \ldots, e_{k}\right)$ ("explicit parallel construct $\circledast_{e x}$ satisfies $P$ ") indexed over all parallel constructs $\circledast_{e x}\left(e_{1}, \ldots, e_{k}\right)$ the predicates $\vdash_{P} E$ ("any parallel construct occurring in $E$ satisfies $P$ ") and $E \vdash_{P} e$ ("any parallel constructs occurring in $E$ and $e$ satisfy $P$ ") are defined as the homomorphic extensions of 
$P_{\circledast_{e x}}$ by the following rules:

$$
\begin{gathered}
\overline{\vdash_{P}\langle\rangle} \\
\frac{E \vdash_{P} c}{\vdash_{P} E \oplus c} \\
\frac{E \vdash_{P} c \quad E \oplus c \vdash_{P} e}{E \vdash_{P} c \Theta e} \\
\frac{\vdash_{P} E \quad E \vdash_{P} e_{1}, \ldots, E \vdash_{P} e_{k} \quad P_{\circledast_{e x}}\left(E, e_{1}, \ldots, e_{k}\right)}{E \vdash_{P} \circledast_{e x}\left(e_{1}, \ldots, e_{k}\right)}
\end{gathered}
$$

The first three rules are very obvious with respect to the intuitive meaning of the predicates given above. The last rule is more subtle. Note that it is possible that $k=0$, for example take $\circledast_{e x} 0=$ prim, and hence the condition $\vdash_{p} E$ is indispensable. Furthermore, the last two rules justify the use of the adjectives "sequential" and "parallel".

\subsection{Closed Expressions}

The first semantic assertion to be defined is the one of closed expressions, i.e. closed texts and closed contexts, relative to an environment. Figuratively speaking, a text or a context is closed relative to an environment if all applied occurrences of identifiers have a corresponding binding in the environment. Other occurrences of identifiers, e.g. as selector names in products, do not require a binding.

The predicate $c l_{\circledast_{e x}}\left(E, e_{1}, \ldots, e_{k}\right)$ checks, in case $\circledast_{e x}$ denotes an applied occurrence of an identifier, whether it has a binding in the environment $E$. The predicate is defined by the following equations, where it was chosen to write $c l_{E}\left(\circledast_{e x}\left(e_{1}, \ldots, e_{k}\right)\right)$ for $c l_{\circledast_{e x}}\left(E, e_{1}, \ldots, e_{k}\right)$ :

$$
\begin{aligned}
& c l_{E}(x)=\text { true if }(E \uparrow x) \text { is defined } \\
& \left.c l_{E} \text { (import } p\right)=\text { true if }(E \backslash p) \text { is defined } \\
& c l_{E}(c[X=: Y])=\operatorname{binds}_{E}(c, X) \\
& c l_{E}\left(\circledast_{e x}\left(e_{1}, \ldots, e_{k}\right)\right)=\text { true if } \circledast_{e x}\left(e_{1}, \ldots, e_{k}\right) \text { denotes neither } x \\
& \text { nor import } p \text {, nor } c[X=: Y]
\end{aligned}
$$

Closed environments $\vdash_{c l} E$ and closed expressions $E \vdash_{c l} e$ are defined as the homomorphic extensions of $c l_{E}$. The contexts from Figs. 3 and 4 are closed.

\subsection{Reduction of Expressions}

This section will introduce an equivalence relation on expressions. The intention is to identify those expressions that are syntactically different, although intuitively they have the same constructive content. There are several ways to introduce such an equivalence. In this presentation, it is defined in an operational way by defining a process, called reduction, to transform expressions into a normal form.

The reduction relation is defined as the reflexive and transitive closure of a "single-step reduction". The single-step reduction of Deva is denoted by $\triangleright^{\prime}$ where reduction takes place relative to an environment to resolve the binding of identifiers, i.e. $E \vdash e_{1} \triangleright^{1} e_{2}$ asserts that the expression $e_{1}$ reduces in a single 
step to $e_{2}$ relative to the environment $E$. The single-step reduction is specified by the following set of rules grouped into axioms and structural rules. The following axioms define the elementary reduction of texts:

A defined identifier may be replaced by its definiens, i.e.

$$
E \vdash x \triangleright^{1} t, \quad \text { if } E \uparrow x=x:=t .
$$

The following two rules define the instantiation of the $n$-th declaration of an abstraction:

$$
\begin{gathered}
E \vdash\left[x: t_{1} \vdash t_{2}\right](1:=t) \triangleright^{1}\left[x:=t \vdash t_{2}\right], \\
E \vdash\left[x: t_{1} \vdash t_{2}\right](n+1:=t) \triangleright^{1}\left[x: t_{1} \vdash t_{2}(n:=t)\right] .
\end{gathered}
$$

Note that in contrast to $\lambda$-calculus no external substitution operation is introduced, but the internal notion of definition is used, leading to an axiom which resembles a graph reduction step. Also note that no applicability condition, i.e. that the type of $t$ matches $t_{1}$, is imposed on axiom R2. This illustrates once again that at this point of the language definition, type constraints are of no concern. Further note that texts containing faulty applications, e.g. $E \vdash \operatorname{prim}\left(2:=t_{2}\right)$, remain irreducible. In fact, the semantic relation of "validity" (cf. 2.11) excludes these and many other kinds of "errors" in expressions. There will never be any need to reduce such expressions, it is thus sufficient to consider reduction of "well-behaved" situations only.

Definitions inside abstractions can be removed, given that they are completely unfolded. Joined contexts inside abstractions can be split:

$$
\begin{gathered}
E \vdash\left[x:=t_{1} \vdash t_{2}\right] \triangleright^{1} t_{2}, \quad \text { if } E \vdash_{c l} t_{2} \\
E \vdash[\text { context } p:=c \vdash t] \triangleright^{\prime} t, \quad \text { if } E \vdash_{c l} t \\
E \vdash\left[\llbracket c_{1} ; c_{2} \rrbracket \vdash t\right] \triangleright^{\prime}\left[c_{1} \vdash\left[c_{2} \vdash t\right]\right]
\end{gathered}
$$

Reduction detaches type assertions from judgements:

$$
E \vdash t_{1} \therefore t_{2} \triangleright^{\prime} t_{1}
$$

The following axioms define the (obvious) operational semantics of projection and product modification:

$$
\begin{gathered}
E \vdash\left\langle x_{1}:=t_{1}, \ldots, x_{n}:=t_{n}\right\rceil . i \triangleright^{\prime} t_{i}, \quad \text { if } 1 \leq i \leq n \\
E \vdash\left\langle x_{1}:=t_{1}, \ldots, x_{i}:=t_{i}, \ldots, x_{n}:=t_{n}\right\rangle \text { where } i:=t \\
\triangleright^{\prime}\left\langle x_{1}:=t_{1}, \ldots, x_{i}:=t, \ldots, x_{n}:=t_{n}\right\rangle
\end{gathered}
$$

The next axiom describes the selection of a product component by means of a 1-injection:

$$
\begin{gathered}
E \vdash \operatorname{case}\left\langle x_{1}:={ }^{\wedge} t_{1}|\ldots| x_{i}:=t_{i}|\ldots| x_{n}:={ }^{\wedge} t_{n}\right\rangle \text { of }\left\{y_{1}:=t_{1}^{\prime}, \ldots, y_{n}:=t_{n}^{\prime} \downarrow\right. \\
\triangleright^{1} t_{i}^{\prime}\left(1:=t_{i}\right)
\end{gathered}
$$

The last three axioms describe the operational semantics of the cut operation:

$$
\begin{gathered}
E \vdash\left[x: t_{1} \vdash t_{2}\right] \text { upto } 1 \propto\left[y: t_{3} \vdash t_{4}\right] \text { at } 1 \triangleright^{1}\left[\left[x: t_{1} ; y:=t_{2}\right] \vdash t_{4}\right] \\
E \vdash\left[x: t_{1} \vdash t_{2}\right] \text { upto } m+1 \diamond t_{3} \text { at } 1 \triangleright^{1}\left[x: t_{1} \vdash t_{2} \text { upto } m \diamond t_{3} \text { at } 1\right] \\
E \vdash t_{1} \text { upto } m \diamond\left[x: t_{2} \vdash t_{3}\right] \text { at } n+1 \triangleright^{1}\left[x: t_{2} \vdash t_{1} \text { upto } m \diamond t_{3} \text { at } n\right]
\end{gathered}
$$


Axiom RII describes (ordinary) functional composition between texts, i.e. when replacing $t_{1}$ by $f(x)$ and $t_{2}$ by $g(y)$ then the right hand side becomes [IIx:t $t_{1} ; y:=$ $f(x) \rrbracket \vdash g(y)]$, which can be further reduced to $\left[x: t_{1} \vdash g(f(x))\right]$ based on the axioms already described. Note again that type constraints are of no concern at this point of the definition. Axioms RI2 and RI3 describe a generalization of the cut in a similar way as application was generalized above. Note that in the general case, $t$ upto $m \gg t^{\prime}$ at $n$, these rules enforce that the arguments of $t^{\prime}$ are processed first and the arguments of $t$ last.

The following axioms define the elementary reduction of context operations. The axioms RI4, RI5, and RI6 are counterparts of the axioms RI, R2, and R3 for text reduction:

$$
\begin{array}{r}
E \vdash \operatorname{import} p \triangleright^{1} c, \quad \text { if } E \uparrow p=p:=c \\
E \vdash \llbracket x: t_{1} ; c \rrbracket(1:=t) \triangleright^{1} \llbracket x:=t ; c \rrbracket \\
E \vdash \llbracket x: t_{1} ; c \rrbracket(n+1:=t) \triangleright^{1} \llbracket x: t_{1} ; c(n:=t) \rrbracket
\end{array}
$$

In contrast to text applications, two more axioms are needed to completely define context applications. They are necessary since application is not yet defined for a context consisting of a single declaration, and there is no axiom for contexts which allows removal of (text or context) definitions:

$$
\begin{gathered}
E \vdash\left(x: t_{1}\right)(1:=t) \triangleright^{1} x:=t \\
E \vdash \llbracket x:=t_{1} ; c \rrbracket(n:=t) \triangleright^{1} \llbracket x:=t_{1} ; c(n:=t) \rrbracket \\
E \vdash \llbracket \operatorname{context} p:=c_{1} ; c \rrbracket(n:=t) \triangleright^{1} \llbracket \operatorname{context} p:=c_{1} ; c(n:=t) \rrbracket
\end{gathered}
$$

Nested joined contexts are reduced into right associative form:

$$
E \vdash \llbracket \llbracket c_{1} ; c_{2} \rrbracket ; c_{3} \rrbracket \triangleright^{1} \llbracket c_{1} ; \llbracket c_{2} ; c_{3} \rrbracket \rrbracket
$$

Finally, the renaming operation is defined in the same way as it was already defined in Sec. 2.4 .

$$
\begin{gathered}
E \vdash(X \odot e)[X=: Y] \triangleright^{\prime}(Y \odot e) \\
E \vdash \llbracket X \odot e ; c \rrbracket[Y=: Z] \triangleright^{1} \begin{cases}\llbracket Z \odot e ; c \rrbracket & \text { if } X=Y \\
\llbracket X \odot e ; c[Y=: Z] \rrbracket, & \text { otherwise }\end{cases}
\end{gathered}
$$

The structural rules state that reduction is monotonic with respect to the syntactic structure of texts and contexts. The rules come in two patterns: two rules for sequential constructs and one rule for parallel constructs.

$$
\begin{gathered}
\frac{E \vdash c_{1} \triangleright^{1} c_{2}}{E \vdash c_{1} \Theta e \triangleright^{1} c_{2} \Theta e} \\
\frac{E \oplus c \vdash e_{1} \triangleright^{1} e_{2}}{E \vdash c \ominus e_{1} \triangleright^{1} c \Theta e_{2}} \\
\frac{E \vdash e_{i} \triangleright^{1} e_{i}^{\prime}}{E \vdash \circledast_{e x}\left(e_{1}, \ldots, e_{i}, \ldots, e_{n}\right) \triangleright^{1} \circledast_{e x}\left(e_{1}, \ldots, e_{i}^{\prime}, \ldots, e_{n}\right)}
\end{gathered}
$$

Now, reduction of expressions is specified as the reflexive and transitive closure 
of single-step reduction of expressions:

$$
\begin{gathered}
\overline{E \vdash e \triangleright e} \\
\frac{E \vdash e_{1} \triangleright^{1} e_{2}}{E \vdash e_{1} \triangleright e_{2}} \\
\frac{E \vdash e_{1} \triangleright e_{2} \quad E \vdash e_{2} \triangleright e_{3}}{E \vdash e_{1} \triangleright e_{3}}
\end{gathered}
$$

The properties of reduction, which include confluence and strong normalization under certain restrictions, are presented in detail in section 4.

Illustrations. The first example illustrates the reduction of projections and applications:

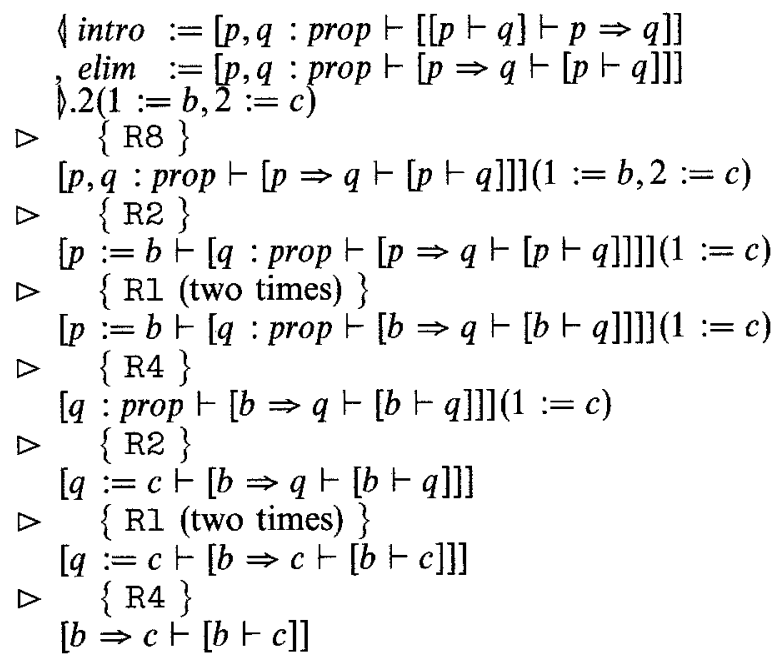

The environment in which this reduction takes place has been omitted. It is assumed to be as within the proof of Syllogism in Fig. 3. In some of the above reduction steps the structural rules (i.e. the rules $\mathrm{RS} j$ ) have been tacitly applied. It is interesting to extend this reduction along the lines of the syllogism proof. Remember that $\left[t_{1} \vdash t_{2}\right]$ is equivalent syntax for $\left[x: t_{1} \vdash t_{2}\right]$, where $x$ is a new identifier:

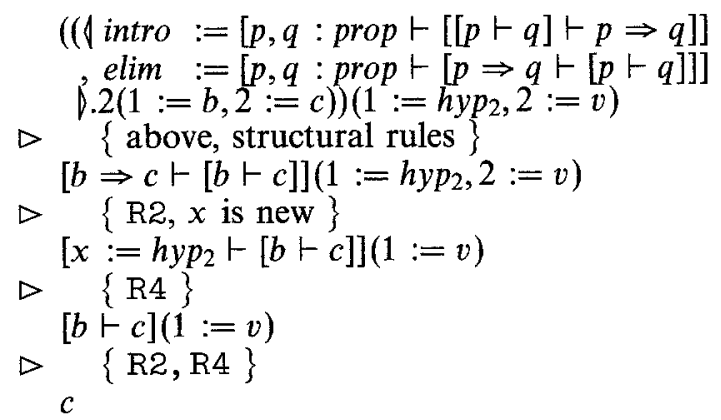


This demonstrates how reduction "consumes" abstractions without checking for type correctness. The example can be extended still further to yield:

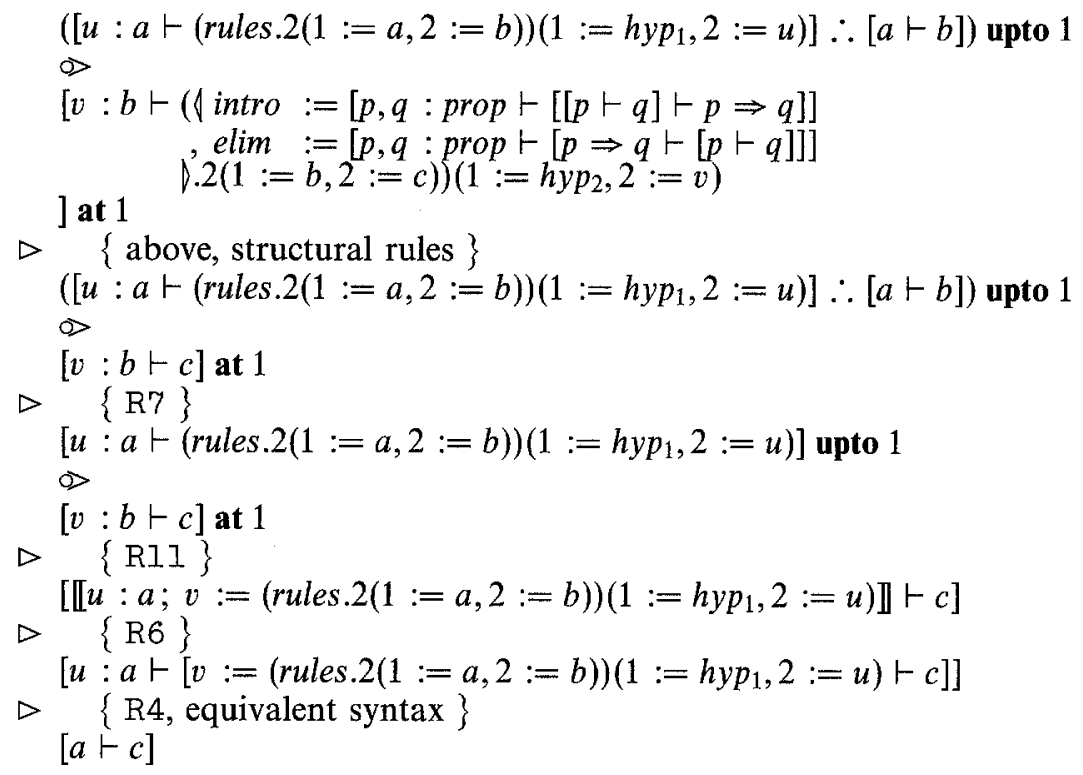

Finally, the reduction of a case distinction is illustrated on a text taken from the simplification example (cf. Fig. 4):

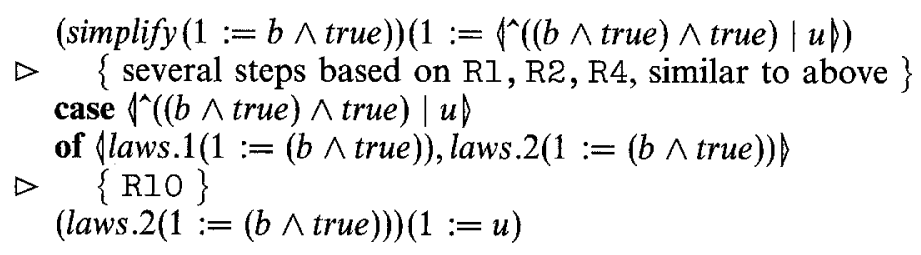

\subsection{Conversion of Expressions}

Conversion is defined to be the equivalence generated by reduction and is denoted by $E \vdash e_{1} \sim e_{2}$ :

$$
\begin{aligned}
& \overline{E \vdash e \sim e} \\
& \frac{E \vdash e_{1} \triangleright e_{2}}{E \vdash e_{1} \sim e_{2}} \\
& \frac{E \vdash e_{1} \sim e_{2}}{E \vdash e_{2} \sim e_{1}} \\
& \frac{E \vdash e_{1} \sim e_{2} E \vdash e_{2} \sim e_{3}}{E \vdash e_{1} \sim e_{3}}
\end{aligned}
$$

Remark. One should be aware, that the axioms R4 and R5 give rise to the fact that conversion, unlike reduction, does not preserve the closedness of expressions, 
i.e. $E \vdash e_{1} \sim e_{2}$ and $E \vdash_{c l} e_{1}$ do not, in general, imply that $E \vdash_{c l} e_{2}$. As a simple example take $t_{1}=[a$ :prim $\vdash a], t_{2}=[b:=c \vdash[a:$ prim $\vdash a]]$, and $E=\langle\rangle$.

Remark. At this point, the time seems right to discuss name-handling in the definition of Deva. Recall that it was assumed that name clashes do not occur in the definitions. In order to justify this assumption, there must be some way to avoid such name-clashes. Two alternative approaches could be pursued at this point to solve the problem. The first one is to define some kind of renaming of bound variables similar to the $\alpha$-conversion of the untyped $\lambda$-calculus. As it turns out however, renaming is difficult to formalize especially since Deva supports the concept of definitions.

A second approach is to use a so-called nameless notation, where each defining occurrence of an identifier is removed and each applied occurrence of an identifier is replaced by a pointer to its binder. One such scheme using naturals as pointers was proposed by de Bruijn [Bru72]. Following this scheme, there is a translation function, say tra, which performs the replacement of identifiers by naturals denoting the the distance from the identifier to its binding. The effect of tra can be illustrated in an example:
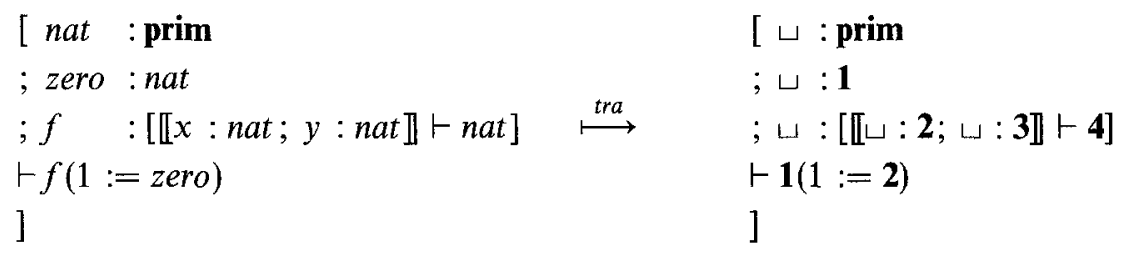

Note that defining occurrences of identifiers are not removed, which would result in invalid syntax, but replaced by a standard identifier $\sqcup$.

This approach is better suited for the definition of Deva, however, tra is somewhat too crude for our purposes since it automatically renames defining occurrences of identifiers, a fact that makes it unsuitable to explicitly deal with operations such as renaming in contexts. A simple solution is to split tra into two functions, i.e. $\operatorname{tr} a=\kappa \circ \tau$. The function $\tau$ translates the applied occurrences of identifiers of a text to their de Bruijn indices, however the defining occurrences remain unchanged.

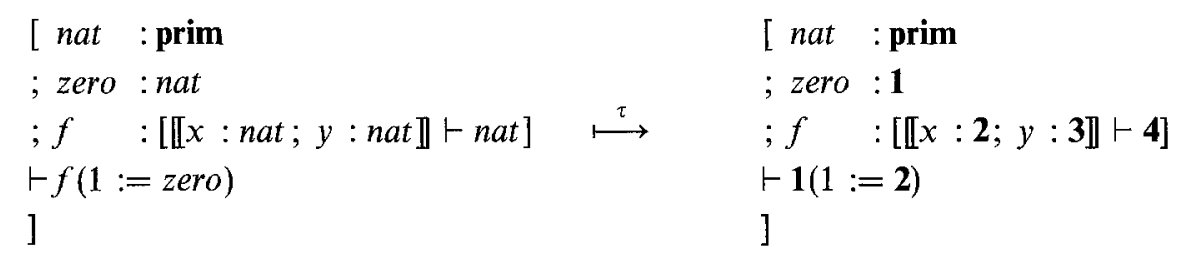

Note that, when restricted to closed expressions, $\tau$ is a bijection, comparable to the name analysis in compiler construction. However, the symbol table is not kept outside but build into the program text. The function $\kappa$ replaces the remaining occurrences of identifiers. 


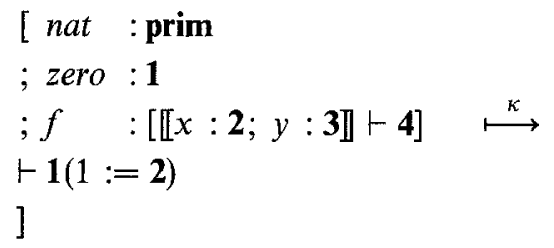

$$
\begin{aligned}
& {[\sqcup: \text { prim }} \\
& ; \sqcup: \mathbf{1} \\
& ; \sqcup:[[\sqcup: \mathbf{2} ; \sqcup: \mathbf{3}] \vdash \mathbf{4}] \\
& \vdash \mathbf{1}(\mathbf{1}:=\mathbf{2}) \\
& ]
\end{aligned}
$$

This refined indexing scheme could have been employed in the definition of Deva from the beginning, but, as de Bruijn pointed out, this notation "is not easy to write or to read for the human reader." To maintain readability, a compromise is proposed. A definition of Deva using de Bruijn indices based on the first translation function $\tau$ is assumed given. Such a definition is a rather technical exercise and can be found in [Web91a]. The definition given in this and the following sections can then be seen as an idealized presentation of this definition, i.e. using names instead of pointers. It is important to understand the consequences of this convention. For example it is not true that

$$
\langle\vdash[x: \text { prim } \vdash x] \sim[y: \operatorname{prim} \vdash y]
$$

since this proposition is an idealized presentation of the underlying proposition

$$
\langle\vdash[x: \operatorname{prim} \vdash 1] \sim[y: \operatorname{prim} \vdash 1]
$$

in which the name difference between the two declarations does matter. However, it is true that

$$
\langle\rangle \vdash \kappa([x: \text { prim } \vdash x]) \sim \kappa([y: \text { prim } \vdash y])
$$

since this is an idealized presentation of the underlying proposition

$$
\langle\rangle \vdash \kappa([x: \operatorname{prim} \vdash 1]) \sim \kappa([y: \operatorname{prim} \vdash 1])
$$

which is clearly true (since $\kappa$ replaces both $x$ and $y$ by $\omega$ ).

Having this in mind, we return to the definition of Deva and introduce a name irrelevant conversion, i.e. conversion modulo $\kappa$. To avoid inconsistencies, we restrict this relation to closed expressions:

$$
\frac{E \vdash_{c l} e_{1} \quad E \vdash_{c l} e_{2} \quad E \vdash \kappa\left(e_{1}\right) \sim \kappa\left(e_{2}\right)}{E \vdash e_{1} \dot{\sim} e_{2}}
$$

\subsection{Type Assignment of Texts}

Besides the operational concept of reduction, the static concept of type assignment is the other fundamental semantic concept of Deva. In Deva only texts have types; the type of a text relative to an environment is again a text expressing the logical result of that text. For example, the type of a text representing a logical derivation is the theorem established by that derivation. The assignment of types to texts is accomplished by the partial function typ : $\mathscr{E} \mathscr{N} \mathscr{V} \times \mathscr{T} \rightarrow \mathscr{T}$. First, the type of the constant prim remains undefined:

$$
\operatorname{typ}_{E}(\text { prim })=\text { undefined }
$$


The typing rules for applied occurrences of identifiers are quite obvious, i.e. replacement either by the declared type or by the type of the definiens:

$$
\operatorname{typ}_{E}(x)= \begin{cases}t & \text { if } E \backslash x=x: t, \\ \operatorname{typ}_{E}(t) & \text { if } E\lceil x=x:=t, \\ \text { undefined } & \text { if } E \backslash x \text { is undefined, }\end{cases}
$$

The typing of a judgement again expresses the fact that the type assertion " $\therefore t_{2}$ " is of no interest at this point of definition:

$$
\operatorname{typ}_{E}\left(t_{1} \therefore t_{2}\right)=\operatorname{typ}_{E}\left(t_{1}\right)
$$

The typing rule for products is very simple. Figuratively speaking, the type of a product of texts is the product of their types. The typing rules for projection and product modification are natural consequences of that view:

$$
\begin{aligned}
\left.\operatorname{typ}_{E}\left(1 x_{1}:=t_{1}, \ldots, x_{n}:=t_{n}\right\rangle\right) & =\left|x_{1}:=\operatorname{typ}_{E}\left(t_{1}\right), \ldots, x_{n}:=\operatorname{typ}_{E}\left(t_{n}\right)\right| \\
\operatorname{typ}_{E}(t . n) & =\operatorname{typ}_{E}(t) . n \\
\operatorname{typ}_{E}\left(t_{1} \text { where } n:=t_{2}\right) & =\operatorname{typ}_{E}\left(t_{1}\right) \text { where } n:=\operatorname{typ}_{E}\left(t_{2}\right)
\end{aligned}
$$

Abstraction and application are typed as follows:

$$
\begin{aligned}
\operatorname{typ}_{E}([c \vdash t]) & =\left[c \vdash \operatorname{typ}_{E \oplus c}(t)\right] \\
\operatorname{typ}_{E}\left(t_{1}\left(n:=t_{2}\right)\right) & =\operatorname{typ}_{E}\left(t_{1}\right)\left(n:=t_{2}\right)
\end{aligned}
$$

A simple example may help to provide some intuition for these two rules. Assume the environment $E=\langle$ nat : prim, $o:$ nat $\rangle$ introducing a type nat with an element $o$ and consider the application $[x:$ nat $\vdash x](1:=o)$. While intuitively one would expect it to be of type nat, according to the formal typing rules its type is not nat but $[x:$ nat $\vdash$ nat $](1:=o)$. However, it is not difficult to see that, based on axioms R1, R2, and R4, this application can be reduced to the expected type nat.

The typing philosophy for sums is similar to that of products when sums are viewed as incomplete products. The indices of the incompletely presented components are just decremented:

$$
\begin{aligned}
& \operatorname{typ}_{E}\left(\left\{x_{1}:=t_{1}|\ldots| x_{n}:=t_{n}\right\rangle\right) \\
& \quad=\left\{x_{1}:=\operatorname{typ}_{E}\left(t_{1}\right)|\ldots| x_{n}:=\operatorname{typ}_{E}\left(t_{n}\right)\right\rangle \\
& \operatorname{typ}_{E}\left(\left\langle x_{1}:={ }^{k+1} t_{1}|\ldots| x_{i}:=t_{i}|\ldots| x_{n}:={ }^{k+1} t_{n}\right\rangle\right) \\
& \left.\quad=\left\{x_{1}:={ }^{k} t_{1}|\ldots| x_{i}:=\operatorname{typ}_{E}\left(t_{i}\right)|\ldots| x_{n}:={ }^{k} t_{n}\right\rangle\right)
\end{aligned}
$$

The typing rule for case distinctions and for cuts are necessary consequences of the above typing philosophy for abstraction and application, since these constructs are reduced to (combinations) of abstractions and applications (as can be seen from the corresponding reduction axioms):

$$
\begin{aligned}
\operatorname{typ}_{E}\left(\operatorname{case} t_{1} \text { of } t_{2}\right) & =\text { case } t_{1} \text { of } \operatorname{typ}_{E}\left(t_{2}\right) \\
\operatorname{typ}_{E}\left(t_{1} \text { upto } n \gg t_{2} \text { at } m\right) & =t_{1} \text { upto } n \gg \operatorname{typ}_{E}\left(t_{2}\right) \text { at } m
\end{aligned}
$$

Illustrations. The first example illustrates type assignment for a subexpression of Fig. 3 (for clarity, the environment is omitted).

$$
=\quad \begin{aligned}
& \operatorname{typ}(\text { rules. } 2(1:=b, 2:=c)) \\
& \text { TY8 twice }\}
\end{aligned}
$$




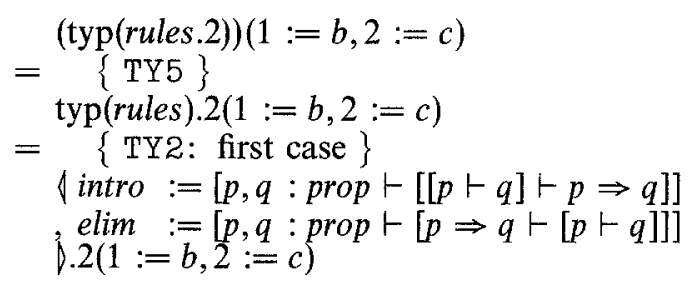

As illustrated in section 2.7 , the latter text reduces to $[a \Rightarrow b \vdash[a \vdash b]]$. It therefore follows that

$$
\operatorname{typ}(\text { rules.2(1:=b,2:=c)) } \sim[b \Rightarrow c \vdash[b \vdash c]]
$$

Extending the example along the lines followed in Sec. 2.7 yields

$$
\text { typ }((\text { rules.2(1:=b,2:=c))(1:=hyp } 2,2:=v)) \sim c
$$

A further extension yields:

$$
\begin{aligned}
& \operatorname{typ}\left(\left(\left[u: a \vdash(\text { rules. } 2(1:=a, 2:=b))\left(1:=\text { hyp }_{1}, 2:=u\right)\right]\right) \therefore[a \vdash b] \text { upto } 1\right. \\
& \left.\circlearrowleft\left(\left[v: b \vdash(\text { rules. } 2(1:=b, 2:=c))\left(1:=\text { hyp }_{2}, 2:=v\right)\right] \therefore[b \vdash c]\right) \text { at } 1\right) \\
& =\{\mathrm{TY} 12\} \\
& \left(\left[u: a \vdash(\text { rules } 2(1:=a, 2:=b))\left(1:=h y p_{1}, 2:=u\right)\right]\right) \therefore[a \vdash b] \text { upto } 1 \\
& =\quad \begin{array}{c}
\operatorname{typ}\left(\left[v: b \vdash(\text { rules } 2(1:=b, 2:=c))\left(1:=h_{y p}, 2:=v\right)\right] \therefore[b \vdash c]\right) \text { at } 1 \\
\operatorname{TY} 3\}
\end{array} \\
& \left(\left[u: a \vdash(\text { rules } 2(1:=a, 2:=b))\left(1:=h y p_{1}, 2:=u\right)\right]\right) \therefore[a \vdash b] \text { upto } 1 \\
& \text { os typ }([v: b \vdash(\text { rules } 2(1:=b, 2:=c))(1:=\text { hyp } 2,2:=v)]) \text { at } 1 \\
& =\{\mathrm{TY} 7\} \\
& \left(\left[u: a \vdash(\text { rules } 2(1:=a, 2:=b))\left(1:=h_{y p}, 2:=u\right)\right] \therefore[a \vdash b]\right) \text { upto } 1 \\
& \text { o }[v: b \vdash \operatorname{typ}((\text { rules. } 2(1:=b, 2:=c))(1:=\text { hyp } 2,2:=v))] \text { at } 1 \\
& =\{\text { TY8 four times } \\
& \left(\left[u: a \vdash(\text { rules } 2(1:=a, 2:=b))\left(1:=h y p_{1}, 2:=u\right)\right] \therefore[a \vdash b]\right) \text { upto } 1 \\
& \text { o> }\left[v: b \vdash(\operatorname{typ}(\text { rules. } 2)(1:=b, 2:=c))\left(1:=h y p_{2}, 2:=v\right)\right] \text { at } 1 \\
& =\{\mathrm{TY} 5\} \\
& \left(\left[u: a \vdash(\text { rules } 2(1:=a, 2:=b))\left(1:=h y p_{1}, 2:=u\right)\right] \therefore[a \vdash b]\right) \text { upto } 1 \\
& \left.\infty\left[v: b \vdash(\operatorname{typ}(\text { rules }) .2(1:=b, 2:=c))\left(1:=\text { hyp }_{2}, 2:=v\right)\right] \text { at } 1\right) \\
& =\{\text { TY2: first case }\} \\
& \left(\left[u: a \vdash(\text { rules } 2(1:=a, 2:=b))\left(1:=h y p_{1}, 2:=u\right)\right] \therefore[a \vdash b]\right) \text { upto } 1 \\
& \text { D } \\
& {[v: b \vdash(\{\text { intro }:=[p, q: \text { prop } \vdash[[p \vdash q] \vdash p \Rightarrow q]]} \\
& \text { ] at } 1 \\
& \text {, elim }:=[p, q: \text { prop } \vdash[p \Rightarrow q \vdash[p \vdash q]]]
\end{aligned}
$$

As shown in section 2.7, the latter expression reduces to $[a \vdash c]$. In other words, the type of the cut in Fig. 3 behaves as required by the judgement.

Remark. Note that, in Deva texts, their types, the types of their types etc. are not given in separate notations but are syntactically identical. This is justified since the given operations of typing and reduction have the same properties on all these levels, thus suggesting introducing a single common data type for these entities. While this leads to a simple syntactic system, the role of a text, i.e. whether it is an element, type, type of type etc., must be determined from the context. 


\subsection{Auxiliary Semantic Predicates for Validity}

In the next section, the notion of validity is defined: a text or a context is valid if certain type constraints are satisfied. The definition of these type constraints uses some auxiliary semantic predicates which are defined in this section.

First, the name-irrelevant conversion is used to define the semantic predicate 'domain'. Figuratively speaking, a text $t_{2}$ is an $n$-th domain of a text $t_{1}$ relative to an environment $E$, denoted by $n-\operatorname{dom}_{E}\left(t_{1}, t_{2}\right)$, if the $n$-th "argument position" of $t_{1}$ has a type convertible to $t_{2}$. This notion is made precise by the following rules:

$$
\begin{aligned}
& \frac{E \vdash \operatorname{typ}_{E}\left(t_{1}\right) \dot{\sim}\left[x: t_{2} \vdash t_{3}\right]}{1-\operatorname{dom}_{E}\left(t_{1}, t_{2}\right)} \\
& \frac{E \vdash t_{1} \dot{\sim}\left[x: t_{2} \vdash t_{3}\right]}{1-\operatorname{dom}_{E}\left(t_{1}, t_{2}\right)}
\end{aligned}
$$

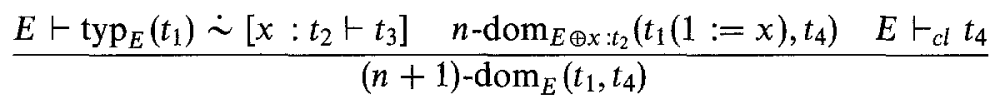

$$
\begin{aligned}
& \frac{E \vdash t_{1} \dot{\sim}\left[x: t_{2} \vdash t_{3}\right] \quad n-\mathrm{dom}_{E \oplus x: t_{2}}\left(t_{3}, t_{4}\right) \quad E \vdash_{c l} t_{4}}{(n+1)-\operatorname{dom}_{E}\left(t_{1}, t_{4}\right)}
\end{aligned}
$$

Remarks. One might wonder why it is necessary to have rules DT2 and DT4 in addition to rules DTI and DT3. This is due to the fact that a text like $[x$ : prim $\vdash$ prim] is perfectly valid but cannot be typed. Thus its first domain can be determined by rule DT2 but not by rule DTI. Note that this introduces the possibility that the first domain of texts like $[x:$ prim $\vdash x]$ can be determined in two different ways. However, the domains determined are unique up to name-free conversion, i.e. it can be shown in general that $n-\operatorname{dom}_{E}\left(t, t_{1}\right)$ and $n-\operatorname{dom}_{E}\left(t, t_{2}\right)$ together imply that $E \vdash t_{1} \dot{\sim} t_{2}$. The third premise in the rules DT3 and DT4 is necessary, since domains which depend on unknown arguments make no sense, as e.g. in the case of the second domain of the text $[x:$ prim; $y: x \vdash y]$. Note that in rule DT3, $t_{1}$ is applied to an abstract argument $x$ in order to get hold of its subsequent domains. In rule DT4 this application could be simplified thanks to the property $E \vdash t_{1}(1:=x) \sim t_{3}$.

Illustrations. Based on the illustrations given in sections 2.7 and 2.9, it is not difficult to infer that e.g. 1-dom(rules.2, prop), 2-dom(rules.2, prop), 1-dom(rules.2(1:=a,1:=b),p $\Rightarrow q)$, and 2-dom(rules.2(1:=a,1:=b),p).

The semantic predicate 'domain' is not only defined between two texts but also between a context and a text. A text $t$ is an $n$-th domain of a context $c$ relative to an environment $E$, denoted by $n$ - $\operatorname{dom}_{E}(c, t)$, if the $n$-th declaration of $c$ has a type convertible to $t$. This notion is made precise by the following rules:

$$
\begin{gathered}
\frac{E \vdash c \dot{\sim} x: t}{1-\operatorname{dom}_{E}(c, t)} \\
\frac{E \vdash c \dot{\sim} \llbracket x: t ; c_{1} \rrbracket}{1-\operatorname{dom}_{E}(c, t)} \\
\frac{E \vdash c \dot{\sim} \llbracket x: t_{1} ; c_{1} \rrbracket \quad n-\operatorname{dom}_{E \oplus x: t_{1}}\left(c_{1}, t_{2}\right) \quad E \vdash_{c l} t_{2}}{(n+1)-\operatorname{dom}_{E}\left(c, t_{2}\right)}
\end{gathered}
$$




$$
\frac{E \vdash c \dot{\sim} \llbracket x:=t_{1} ; c_{1} \rrbracket n-\operatorname{dom}_{E \oplus x:=t_{1}}\left(c_{1}, t_{2}\right) \quad E \vdash_{c l} t_{2}}{n-\operatorname{dom}_{E}\left(c, t_{2}\right)}
$$

Remarks. Since contexts are not typed, there is no need of additional rules due to typing issues. On the other hand, since contexts are always non-empty, two rules are necessary to define 1-dom and since definitions cannot be removed from contexts, an additional rule is necessary in order to allow definitions to be skipped when defining $n$-dom.

Somewhat dual to the semantic predicate 'domain', there is the semantic predicate 'range'. Figuratively speaking, a text $t_{2}$ is an $n$-th range of the text $t_{1}$ relatively to an environment $E$, denoted by $n-\operatorname{ran}_{E}\left(t_{1}, t_{2}\right)$, if $t_{2}$ is convertible to the text appearing after the $n$-th argument position of the type of $t_{1}$. This notion is made precise by the following rules:

$$
\begin{gathered}
\frac{E \vdash \operatorname{typ}_{E}\left(t_{1}\right) \dot{\sim}\left[x: t_{2} \vdash t_{3}\right]}{1-\operatorname{ran}_{E}\left(t_{1}, t_{3}\right)} \\
\frac{E \vdash \operatorname{typ}_{E}\left(t_{1}\right) \dot{\sim}\left[x: t_{2} \vdash t_{3}\right] \quad n-\operatorname{ran}_{E \oplus x: t_{2}}\left(t_{1}(1:=x), t_{4}\right) \quad E \vdash_{c l} t_{4}}{(n+1)-\operatorname{ran}_{E}\left(t_{1}, t_{4}\right)}
\end{gathered}
$$

Illustrations. For example, 1-ran(rules.2(1:=a,1:=b), $[p \vdash q])$ or 2-ran(rules.2(1:=a,1:=b),q).

The type constraint of case distinctions involves the predicate 'domain sum'. A text $t_{2}$ is a domain sum of the text $t_{1}$ relative to an environment $E$, denoted by dom-sum ${ }_{E}\left(t_{1}, t_{2}\right)$, if $t_{2}$ is convertible to a sum of texts. These correspond to the first declared types of a product of abstractions yielded by $t_{1}$. This notion is made precise by the following rules:

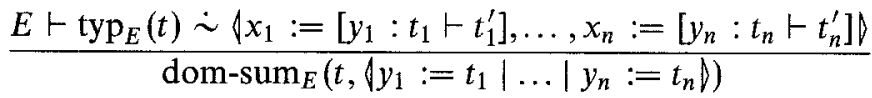

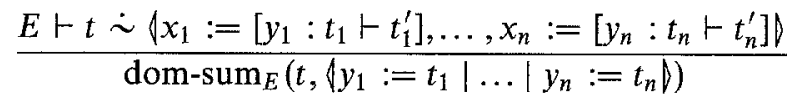

Illustration. A domain sum occurs in Fig. 2 as follows:

$$
\begin{array}{r}
\text { dom-sum(simplify }(1:=(b \wedge \text { true }), 1:=(b \wedge \text { true })) \\
((b \wedge \text { true }) \wedge \text { true } \mid(b \wedge \text { true }) \vee \text { false }))
\end{array}
$$

The type constraints about projection and product modification involve the semantic predicate 'product size'. A text $t$ is a product of size $n$ relative to an environment $E$, denoted by pro-size $_{E}(t, n)$, if $t$ yields a product with $n$ components. This notion is made precise by the following rules:

$$
\begin{aligned}
& \frac{E \vdash \operatorname{typ}_{E}(t) \dot{\sim}\left\{x_{1}:=t_{1}, \ldots, x_{n}:=t_{n}\right\}}{\text { pro-size }_{E}(t, n)} \\
& \frac{E \vdash t \sim\left\langle x_{1}:=t_{1}, \ldots, x_{n}:=t_{n}\right\rangle}{\text { pro-size }_{E}(t, n)}
\end{aligned}
$$

Illustrations. It is easy to see that pro-size(rules, 2) and pro-size(laws, 2). 


\subsection{Validity}

Now, the central semantic assertion of validity can be defined. An expression $e$, i.e. a text or a context, is valid if each of its constructs satisfies certain (type) constraints. Intuitively, for a text $t$ describing a logical derivation to be valid under an environment $E$ means that the derivation is without gaps and makes proper use of the rules and axioms available in $E$. The predicate $v a l_{\circledast_{e x}}\left(E, e_{1}, \ldots, e_{n}\right)$ describes the validity constraint of the parallel construct $\circledast_{e x}$. For text constructs, it is defined by the following equations in which $\operatorname{val}_{E}\left(\circledast_{e x}\left(e_{1}, \ldots, e_{n}\right)\right)$ represents $v_{a l} l_{\circledast_{e x}}\left(E, e_{1}, \ldots, e_{n}\right)$.

Naturally, the primitive text and all bound identifiers are valid:

$$
\begin{aligned}
\operatorname{val}_{E}(\text { prim }) & =\text { true } \\
\operatorname{val}_{E}(x) & =\text { true, if }(E\lceil x) \text { is defined }
\end{aligned}
$$

An application $t_{1}\left(n:=t_{2}\right)$ is valid if the type of the "argument" $t_{2}$ is an $n$-th domain of the "function" $t_{1}$ :

$$
\operatorname{val}_{E}\left(t_{1}\left(n:=t_{2}\right)\right)=n-\operatorname{dom}_{E}\left(t_{1}, \operatorname{typ}_{E}\left(t_{2}\right)\right)
$$

A judgement $t_{1} \therefore t_{2}$ is valid if the type of the text $t_{1}$ is name-free convertible to the "type tag" $t_{2}$ :

$$
\operatorname{val}_{E}\left(t_{1} \therefore t_{2}\right)=E \vdash \operatorname{typ}_{E}\left(t_{1}\right) \dot{\sim} t_{2}
$$

Component names in products must be disjoint. However, the components need not be all of the same type. Projections and product modifications must not have references exceeding the size of the product they work on:

$$
\begin{aligned}
\operatorname{val}_{E}\left(\left|x_{1}:=t_{1}, \ldots, x_{n}:=t_{n}\right\rangle\right) & =\left(x_{i} \neq x_{j} \text { for } i \neq j\right) \\
\operatorname{val}_{E}(t . n) & =\text { pro-size }_{E}(t, m) \text { for some } t_{3} \text { such that } m \geq n
\end{aligned}
$$

$$
\text { val }_{E}\left(t_{1} \text { where } n:=t_{2}\right)=\text { pro-size }_{E}\left(t_{1}, m\right) \text { where } m \geq n
$$

In particular, it is not excluded that the modification of a product component alters its type.

There is no validity constraint for sums. However, case distinctions must be complete:

$$
\begin{aligned}
& \operatorname{val}_{E}\left(\left|x_{1}:={ }^{k} t_{1}\right| \ldots \mid x_{i}:=\right.\left.\left.t_{i}|\ldots| x_{n}:={ }^{k} t_{n}\right\rangle\right)=\text { true } \\
& \operatorname{val}_{E}\left(\text { case } t_{1} \text { of } t_{2}\right)=\text { dom-sum } \\
& E\left(t_{2}, \operatorname{typ}_{E}\left(t_{1}\right)\right)
\end{aligned}
$$

In a valid cut, the $m$-th range of the first argument of a cut must match the $n$-th domain of the second argument:

$$
v l_{E}\left(t_{1} \text { upto } m \gg t_{2} \text { at } n\right)=n-\operatorname{dom}_{E}\left(t_{2}, t_{3}\right) \text { where } m-\operatorname{ran}_{E}\left(t_{1}, t_{3}\right)
$$

For context constructs, validity is defined by the following equations:

$$
\begin{aligned}
\operatorname{val}_{E}(X \odot e) & =\text { true } \\
\operatorname{val}_{E}(\mathbf{i m p o r t} p) & =\text { true if }(E \nmid p) \text { is defined } \\
\operatorname{val}_{E}(c(n:=t)) & =n-\operatorname{dom}_{E}\left(c, \operatorname{typ}_{E}(t)\right) \\
\operatorname{val}_{E}(c[X=: Y]) & =\operatorname{binds}_{E}(c, X)
\end{aligned}
$$


The first three rules are analogous to the corresponding text rules. The last rule requires a renaming operation to be well-defined.

The validity of an environment $E$, denoted by $\vdash_{v a l} E$, and of an expression $e$ relative to an environment $E$, denoted by $E \vdash_{\text {val }} e$, are defined as the homomorphic extensions of val.

Remarks. Note that val is stronger than $c l$, so that whenever $E \vdash_{v a l} e$ holds then $E \vdash_{c l} e$ holds too.

Illustrations. Both examples of explicit expressions presented in section 2.3 are valid. For example, the validity condition for a core judgement of the syllogism proof (Fig. 3) has been verified in section 2.9 .

\section{Definition of the Implicit Part of Deva}

\subsection{Formation}

The implicit part of Deva is syntactically an extension of the explicit part. The class of texts of the implicit part is specified by the following grammar:

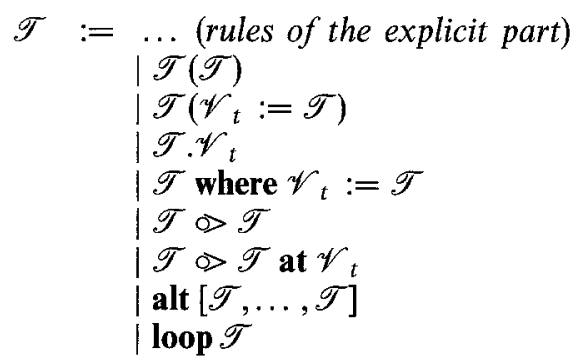

direct application
named application
named projection
named product modification
direct cut
named cut
alternative
iteration

The class of contexts of the implicit part is specified by the following grammar:

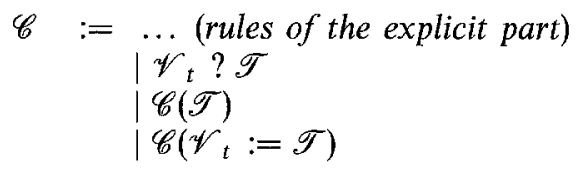

implicit definition
direct context application
named context application

The name conventions from the explicit part are also used in the following discussions. Thus, now $t, c$, and $e$ range over texts, contexts, and expressions of the implicit part.

\subsection{Intended Meaning of the Constructs}

In principle, the valid texts and contexts of the explicit part already constitute a satisfactory means of expression. However, since every construction within an explicitly valid expression must be provided in full detail, the explicit part as such becomes too verbose for practical use. The below operations of the implicit part allows (sometimes quite huge) gaps to be left in developments. Due to the presence of gaps it becomes impossible to impose type constraints on implicit proofs as done for explicit proofs. However, it is quite possible to impose "implicit" validity constraints, by requiring that implicit operations (i.e. operations with gaps) can be "explained" (cf. 3.8) by explicit operations that satisfy type constraints. 
An implicit definition $x$ ? $t$ declares an identifier $x$ to have the type $t$, just like an ordinary declaration $x: t$ would do. The two other context operations of the implicit level are variants of the context application of the explicit level. A direct context application $c(t)$ instantiates the first declaration in $c$ by the text $t$. A named context application $c(x:=t)$ instantiates the declared identifier named $x$ in $c$ by $t$. The two text applications of the implicit level are variants of the text operations of the explicit level, analogous to the context applications. Projections and product modifications are introduced in variants which allow concrete names to be used for selection, instead of natural numbers. The direct cut behaves like the special case of the cut that corresponds to classical functional composition, i.e. $t_{1} \gg t_{2}$ composes a function $t_{1}$ with $t_{2}$ at the first declaration of $t_{2}$. The named cut $t_{1} \gg t_{2}$ at $x$ composes a function $t_{1}$ with $t_{2}$ at the declared identifier named $x$.

However this is not the whole story: All these implicit operations need not satisfy explicit type constraints! Instead, as hinted above, they must be "explainable" using valid explicit operations. Some simple examples may help to illustrate the principle idea behind explaining implicit by explicit expressions. First, assume the environment $\langle x: \operatorname{prim} ; y: x ; f:[z$ ? prim $\vdash[w: z \vdash w]]\rangle$. With respect to this environment, the direct application $f(y)$ is not explicitly valid, since the type $x$ of the argument $y$ does not match the domain $z$ of the first declaration of the function $f$. However, it is implicitly valid because it can be explained by $(f(1:=x))(1:=y)$, which in turn is an explicitly valid expression (assuming $f:[z: \operatorname{prim} \vdash[w: z \vdash w]]$ ). More generally, within a direct application $t_{1}\left(t_{2}\right)$, implicit definitions in $t_{1}$ may become instantiated by additional arguments.

Next, assume the environment $\langle x: \operatorname{prim} ; y:[z$ ? prim $\vdash z] ; f:[w:$ prim $\vdash$ prim] $\rangle$ and consider the direct application $f(y)$. Again, it is not explicitly valid, since the type $[z$ ? prim $\vdash z]$ of $y$ does not conform to the domain prim of $f$. However, this application is implicitly valid because it can be explained by the explicitly valid text $f(1:=y(1:=x))$. Thus, explanation may also take place by instantiating implicit definitions in the argument $t_{2}$ of a direct application $t_{1}\left(t_{2}\right)$.

In addition to omissions of parameters, injections may also be omitted in implicit expressions. Finally, the implicit level introduces two completely new text constructs: The alternative alt $\left[t_{1}, \ldots, t_{n}\right]$ is a function, which when applied to an argument, selects among the texts $t_{i}$ for application. The selection must, of course, conform to the type of the argument and it must be explained by an appropriate case distinction. The iteration $\operatorname{loop} t$ denotes an iterated use of the function $t$ within direct application or direct cuts. It must be explained by an appropriate sequence of direct applications or direct cuts.

\subsection{Environments}

Environments of the implicit part of Deva are a simple extension of environments of the explicit part of Deva. The only difference is that a binding $X$ (o) $e$ can also be an implicit definition $X$ ? e. Given this extended notion of binding, the lookup function $E\lceil e$ is defined as before.

The operation $E \oplus c$ to push a context onto an environment is extended in order to deal with the extended notion of binding and to partially evaluate the new context operations. The first extension consists of generalizing all those rules involving a declaration into rules which may involve an assumption, that is a declaration or an implicit definition. If $x \odot t$ denotes an assumption, i.e. : either 
$x: t$ or $x$ ? $t$, then this extension consists of replacing $x: t$ by $x \odot t$ in the rules given in section 2.4. The new context operations are the direct context application (C10), and the named context application (C11). The following rules are added to the definition of $E \oplus c$ :

$$
\begin{aligned}
& \frac{E \oplus c=E \oplus\left\langle x: t_{1}\right\rangle \oplus E_{1}}{E \oplus c(t)=E \oplus\langle x:=t\rangle \oplus E_{1}} \\
& \frac{E \oplus c=E \oplus\left\langle x ? t_{1}\right\rangle \oplus c_{1}}{E \oplus c(t)=E \oplus\left\langle x ? t_{1}\right\rangle \oplus c_{1}(t)} \\
& \begin{array}{c}
E \oplus c=E \oplus\left\langle x:=t_{1}\right\rangle \oplus c_{1} \\
\hline E \oplus c(t)=E \oplus\left\langle x:=t_{1}\right\rangle \oplus c_{1}(t)
\end{array} \\
& \frac{E \oplus c=E \oplus\left\langle\text { context } p:=c_{2}\right\rangle \oplus c_{1}}{E \oplus c(t)=E \oplus\left\langle\text { context } p:=c_{2}\right\rangle \oplus c_{1}(t)} \\
& \frac{E \oplus c=E \oplus\left\langle x \odot t_{1}\right\rangle \oplus E_{1}}{E \oplus c(x:=t)=E \oplus\langle x:=t\rangle \oplus E_{1}} \\
& \frac{E \oplus c=E \oplus\left\langle y \odot t_{1}\right\rangle \oplus c_{1} \quad y \neq x}{E \oplus c(x:=t)=E \oplus\left\langle y \odot t_{1}\right\rangle \oplus c_{1}(x:=t)} \\
& \frac{E \oplus c=E \oplus\left\langle y:=t_{1}\right\rangle \oplus c_{1}}{E \oplus c(x:=t)=E \oplus\left\langle y:=t_{1}\right\rangle \oplus c_{1}(x:=t)} \\
& \frac{E \oplus c=E \oplus\left\langle\text { context } p:=c_{2}\right\rangle \oplus c_{1}}{E \oplus c(x:=t)=E \oplus\left\langle\text { context } p:=c_{2}\right\rangle \oplus c_{1}(x:=t)}
\end{aligned}
$$

As can be seen from the above rules, a direct context application $c(t)$ amounts to the replacement of the first declaration $x: t_{1}$ in $c$ by the definition $x:=t$ and a context application $c(x:=t)$ amounts to the replacement of the first assumption $x \odot t_{1}$ in $c$ by the definition $x:=t$.

\subsection{Homomorphic Extension, Closed Expressions}

The notion of homomorphic extension is extended in the sense that parallel constructs now also include all the new operations of the implicit part. Let $\mathscr{P} \mathscr{A} \mathscr{R}_{\text {im }}$ denote the set of parallel phrases of the implicit part specified by the grammar:

$$
\begin{aligned}
\mathscr{P} \mathscr{A} \mathscr{R}_{i m}:= & \mathscr{T}_{1}\left(\mathscr{T}_{2}\right)\left|\mathscr{T}_{1}\left(\mathscr{V}_{t}:=\mathscr{T}_{2}\right)\right| \\
& \mathscr{T}_{1} \cdot \mathscr{V}_{t} \mid \mathscr{T}_{1} \text { where } \mathscr{V}_{t}:=\mathscr{T}_{2} \mid \\
& \mathscr{T}_{1} \otimes \mathscr{T}_{1} \mid \mathscr{T}_{1} \oslash \mathscr{T}_{2} \text { at } \mathscr{V}_{t} \mid \\
& \text { alt }\left[\mathscr{T}_{1}, \ldots, \mathscr{T}_{k}\right]\left|\operatorname{loop} \mathscr{T}_{1}\right| \\
& \mathscr{V}_{t} ? \mathscr{T}_{1}\left|\mathscr{C}_{1}\left(\mathscr{T}_{2}\right)\right| \mathscr{C}_{1}\left(\mathscr{V}_{t}:=\mathscr{T}_{2}\right) .
\end{aligned}
$$

An element of $\mathscr{P}_{\mathscr{A}} \mathscr{R}_{i m}$ is denoted by $\circledast_{i m}\left(e_{1}, \ldots, e_{k}\right)$. Using this extended notion of homomorphic extension, closed expressions are defined in the same way as in the explicit part. 


\subsection{Extension of Reduction and Explicit Validity}

The notion of reduction is extended in a way that is similar to the way $E \oplus c$ was extended above, i.e. $x: t$ is replaced by $x \odot t$ in all the axioms defining the elementary reduction steps. The structural rules and the closure rules are not changed. Similarly, the auxiliary semantic predicates from the explicit part are extended by replacing $x: t$ everywhere by $x \odot t$. This implies an extension of explicit validity to cover implicit definitions, treated in exactly the same way as declarations.

\subsection{Auxiliary Semantic Predicates for Implicit Validity}

The implicit part has introduced a number of constructs that are slight variants of already existing explicit constructs, i.e. direct application, named application, and so on. In this section, a number of auxiliary relations are specified and then used to relate these constructs of the implicit part to constructs of the explicit part.

In the explicit part, the auxiliary relation $n$-th domain was already defined (cf. 2.10). Several variations of this relation are introduced in the implicit part. The first such variation is the relation declaration position. Figuratively speaking, a text $t$ has declaration position $n$ relative to an environment $E$, denoted by dec-pos $_{E}(t, n)$, if the $n$-th assumption in $t$ is a declaration, i.e. it is not an implicit definition. This is made precise by the following rules:

$$
\begin{aligned}
& \frac{E \vdash \operatorname{typ}_{E}(t) \dot{\sim}\left[x: t_{1} \vdash t_{2}\right]}{{\operatorname{dec}-\operatorname{pos}_{E}(t, 1)}(t)} \\
& \frac{E \vdash t \dot{\sim}\left[x: t_{1} \vdash t_{2}\right]}{\operatorname{dec}_{-p_{0}}(t, 1)}
\end{aligned}
$$

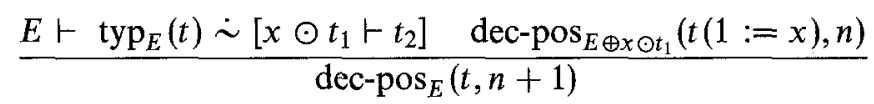

$$
\begin{aligned}
& \frac{E \vdash t \dot{\sim}\left[x \odot t_{1} \vdash t_{2}\right] \quad \text { dec-pos }}{\operatorname{dec}_{E \oplus x \odot t_{1}}\left(t_{2}, n\right)}
\end{aligned}
$$

Note that the structure of the rules is similar to that of the rules for $n-t h$ domain.t

The minimal declaration position, denoted by $\min -\mathrm{dec}_{-} \operatorname{pos}_{E}(t, n)$, is defined as a predicate that holds if and only if $n$ is the smallest integer $m$ such that dec-pos ${ }_{E}(t, m)$ holds. As will be seen later, this predicate allows a precise relation between e.g. the direct application of the implicit part to the application of the explicit part.

Also, there exist analogous variants of the above relations for contexts, denoted by dec-pos ${ }_{E}(c, n)$, and min-dec-pos $\left.(c, n)\right)$. By replacing the two declarations in the above rules by implicit definitions one obtains the rules of the relation implicit definition position, denoted by idef-pos ${ }_{E}(t, n)$, and consequently all the other relations, i.e. min-idef- $\operatorname{pos}_{E}(t, n)$ and the corresponding relations on contexts. All these definitions are quite straightforward and are therefore omitted.

Illustrations. In this and the following illustrations we refer to Figs. 1 and 2. In the context of Fig. 1 it is easy to infer that dec-pos(rules.2,3), dec-pos(rules.2,4), min-dec-pos(rules.2, 3). 
The named applications and named cuts of the implicit part which specify the parameter to be instantiated by giving its name, e.g. $t_{1}\left(x:=t_{2}\right)$, are related to the application and cut of the explicit part by the predicate assumption position. Roughly speaking, a text $t$ has an $x$-assumption position $n$ relative to an environment $E$, denoted by $x$-asm- $\operatorname{pos}_{E}(t, n)$, if the $n$-th assumption in $t$ is an assumption $x \odot t_{1}$. Unfortunately it turns out that there exist cases where the identifier used in the $n$-th assumption differs, depending on whether $t$ or $\operatorname{typ}_{E}(t)$ is considered. For example, take the text

$$
[z:[x: \text { prim } \vdash \text { prim }] \vdash z](1:=[y: \operatorname{prim} \vdash y])
$$

which indeed has either $x$ : prim or $y$ : prim as first assumption position, depending on whether its type is considered or not. Such problematic cases are excluded by the predicate asm-unique $E_{E}(t)$ which is true if and only if $E \vdash t \sim$ $\left[x \odot t_{1} \vdash t_{2}\right]$ and $E \vdash \operatorname{typ}_{E}(t) \sim\left[y \odot t_{1}^{\prime} \vdash t_{2}^{\prime}\right]$ implies that $x=y$. This predicate can be algorithmically realized by simply inspecting the first assumptions in the "normal forms" (cf. 4.7) of $t$ and $\operatorname{typ}_{E}(t)$. Now, the predicate $x$-assumption position can be specified by the following rules:

$$
\begin{aligned}
& \frac{E \vdash \operatorname{typ}_{E}(t) \sim\left[x \odot t_{1} \vdash t_{2}\right] \quad \text { asm-unique }_{E}(t)}{x \text {-asm-pos }} \\
& \frac{E \vdash t \sim\left[x \odot t_{1} \vdash t_{2}\right] \quad \text { asm-unique }_{E}(t)}{x \text {-asm-pos } E} \\
& \frac{E \vdash \operatorname{typ}_{E}(t) \sim\left[y \odot t_{1} \vdash t_{2}\right] \quad x \text {-asm-pos } \operatorname{apy}_{E \oplus t_{1}}(t(1:=y), n)}{x-\operatorname{asm}-\operatorname{pos}_{E}(t, n+1)} \\
& \frac{E \vdash t \sim\left[y \odot t_{1} \vdash t_{2}\right] \quad x \text {-asm-pos }{ }_{E \oplus y \odot t_{1}}\left(t_{2}, n\right)}{x \text {-asm-pos }{ }_{E}(t, n+1)}
\end{aligned}
$$

Once again, the minimal assumption position, denoted by $x$-min-asm-pos ${ }_{E}(t, n)$, is defined as a predicate that holds if and only if $n$ is the smallest integer $m$ such that $x$-min-asm-pos $(t, m)$ holds. Again, there exist variants of this predicate for contexts (these are denoted by $x$-asm-pos $(c, n)$ and $x$-min-asm-pos $\left.\operatorname{pos}_{E}(c, n)\right)$. Their definition is obvious and therefore omitted.

Illustrations. In the context of Fig. 2 we have that $p$-asm-pos(rules.2,1), $q$-asm-pos(rules.2,2), and p-min-asm-pos(rules.2, 1).

The product operations of the implicit part which specify the interesting component by giving its name, e.g. t.x, are related to the product operations of the explicit part by the predicate component position. Roughly speaking, a text $t$ has an $x$-component position at $n$ relative to an environment $E$, denoted by $x$-com-pos ${ }_{E}(t, n)$, if $t$ yields a product $\left\langle x_{1}:=t_{1}, \ldots, x:=t_{n}, \ldots, x_{m}:=t_{m}\right\rangle$. Note that similar to assumption names it is well possible that component names can change, depending on whether a text or its type is considered. For example take the text,

$$
[z:\langle x:=\text { prim, } y:=\operatorname{prim}\rangle \vdash z](1:=\langle y:=w, x:=w \downarrow)
$$

(where $w:$ prim) which has either $x$ or $y$ as its first component name, depending on whether its type is considered or not. Again, there is a predicate com-unique ${ }_{E}(t)$ to enforce the uniqueness of the component names of $t$ and typ $\operatorname{pa}_{E}(t)$. It is true if and only if $E \vdash t \sim \downarrow \ldots, x:=t, \ldots\rangle$ and $E \vdash \operatorname{typ}_{E}(t) \sim \uparrow \ldots, y:=t^{\prime}, \ldots \downarrow$ implies that $x=y$. The predicate $x$-component position is now made precise by 
the following rules:

$$
\begin{gathered}
E \vdash \operatorname{typ}_{E}(t) \sim\left\langle x_{1}:=t_{1}, \ldots, x:=t_{n}, \ldots, x_{m}:=t_{m}\right\rangle \\
\text { com-unique }_{E}\left(\operatorname{typ}_{E}(t)\right) \\
\begin{array}{c}
x \text {-com-pos } \\
E
\end{array}(t, n) \\
E \vdash t \sim\left\langle x_{1}:=t_{1}, \ldots, x:=t_{n}, \ldots, x_{m}:=t_{m}\right\rangle \\
\text { com-unique }_{E}(t) \\
x \text {-com-pos } \text { com }_{E}(t, n)
\end{gathered}
$$

Illustrations. and-com-pos(laws, 1), or-com-pos(laws, 2).

\subsection{Explicitation}

A central semantic relation of the implicit part describes the "explicitation", denoted by $E \vdash e_{1} \sqsupseteq e_{2}$, of an expression $e_{1}$ by an expression $e_{2}$ under an environment $E$. Intuitively $e_{1}$ and $e_{2}$ both are explicitly valid except for one operation, that may be either implicit or explicit, and $e_{2}$ is closer to explicit validity than $e_{1}$.

The laws specifying explicitation come in several groups. The first group of operation explanation rules describes the direct assignment of an explicit construct to an implicit construct. The first four rules deal with applications and projections. These rules make use of the auxiliary predicates just introduced (cf. 3.6) to formalize the intended meaning (cf. 2.2) of those implicit constructs that are close variants of existing constructs of the explicit part:

$$
\begin{gathered}
\frac{\min -\text { dec-pos }_{E}(e, n)}{E \vdash e(t) \sqsupseteq e(n:=t)} \\
\frac{x-\min -\operatorname{asm}_{-p_{E}}(e, n)}{E \vdash e(x:=t) \sqsupseteq e(n:=t)} \\
\frac{x \text {-com-pos }}{E \vdash t . x \sqsupseteq t . n} \\
x \text {-com-pos }\left(t_{1}, n\right) \\
\hline E \vdash t_{1} \text { where } x:=t_{2} \sqsupseteq t_{1} \text { where } n:=t_{2}
\end{gathered}
$$

Remark. Explanation will be defined by applying explicitation recursively on the structure of fully implicit expressions. Therefore in these rules it can be assumed that the arguments of $\circledast_{i m}$ have been already explained, i.e. they are explicitly valid. Therefore, it makes sense to use the auxiliary predicates.

The rules for cuts are quite similar, except that the upto parameter is deliberately chosen to be any natural number $m$ for which an $m$-th range exists in the first argument:

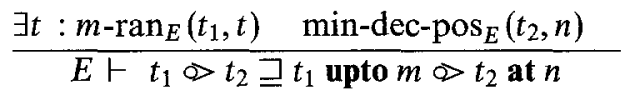

$$
\begin{aligned}
& \frac{\exists t: m-\operatorname{ran}_{E}\left(t_{1}, t\right) \quad x \text {-min-asm-pos }{ }_{E}\left(t_{2}, n\right)}{E \vdash t_{1} \triangleright t_{2} \text { at } x \sqsupseteq t_{1} \text { upto } m \triangleright t_{2} \text { at } n}
\end{aligned}
$$

The last rule shows how an alternative can be explained by a case distinction, in 
case all its components start with a declaration as first domain:

$$
\begin{gathered}
\frac{\operatorname{dec}_{-\operatorname{pos}_{E}}\left(t_{i}, 1\right) \quad 1-\operatorname{val}-\operatorname{dom}_{E}\left(t_{i}, t_{i}^{\prime}\right)}{E \vdash \text { alt }\left[t_{1}, \ldots, t_{n}\right]} \\
\sqsupseteq\left[x:\left|y:=t_{1}^{\prime}\right| \ldots\left|y:=t_{n}^{\prime}\right| \vdash \operatorname{case} x \text { of }\left\{y:=t_{1}, \ldots, y:=t_{n}\right\rangle\right]
\end{gathered}
$$

Note that these rules cannot guarantee the validity constraints of the explicit construct by which the implicit construct has been explained. In fact, there still remain gaps in the explicit operation and therefore these constraints are usually not satisfied. The purpose of the next group of rules is to show how these gaps can be "filled" such that the constraints are satisfied. The first rule describes the extraction of implicitly defined parameters from the arguments of an implicit construct. The notation $n$-val-dom $E(e, t)$ is shorthand for $n-\operatorname{dom}_{E}(e, t), E \vdash_{\text {val }} t$. The predicate $x$-free is defined below.

$$
\begin{gathered}
\begin{array}{c}
\text { idef-pos } \\
E
\end{array}\left(e_{i}, n\right) \quad x \text {-asm-pos }\left(e_{i}, n\right) \\
\frac{n \text {-val-dom }}{E}\left(e_{i}, t\right) \quad x \text {-free }\left(i, \circledast_{i m}\left(e_{1}, \ldots, e_{k}\right)\right) \\
E \vdash \circledast_{i m}\left(e_{1}, \ldots, e_{i}, \ldots, e_{k}\right) \\
\sqsupseteq\left[x ? t \vdash \circledast_{i m}\left(e_{1}, \ldots, e_{i}(n:=x), \ldots, e_{k}\right)\right]
\end{gathered}
$$

Remark. Note that the extracted implicit definition $x$ ? $t$ is logically "connected" with $e_{i}$ via the application $e_{i}(n:=x)$ whose explicit validity follows easily from the preconditions of the rule.

The condition $x$-free $\left(i, \circledast_{i m}\left(e_{1}, \ldots, e_{n}\right)\right)$ serves to prohibit certain paradoxical cases in which the extraction of an implicit definition of an identifier $x$ from a particular argument of $\circledast_{i m}$ would result in a clash with the meaning of $\circledast_{i m}$. For example, the named application $\left[x \quad t_{1} \vdash t_{2}\right]\left(x:=t_{3}\right)$ should be explicitated into the application $\left[x ? t_{1} \vdash t_{2}\right]\left(1:=t_{3}\right)$ only and not into the abstraction $\left[x ? t_{1} \vdash\right.$ $\left.\left(t_{2}(1:=x)\right)\left(x:=t_{3}\right)\right]$. The condition is defined as follows:

$$
\begin{aligned}
x \text {-free }\left(1, t_{1}\left(x:=t_{2}\right)\right) & =\text { false } \\
x-\text { free }\left(2, t_{1} \diamond t_{2} \text { at } x\right) & =\text { false } \\
x \text {-free }(1, c(x:=t)) & =\text { false } \\
x \text {-free }\left(i, \circledast_{i m}\left(e_{1}, \ldots, e_{n}\right)\right) & =\text { true }
\end{aligned}
$$

otherwise

In order to allow multiple extractions of implicit definitions, the following monotonicity rule for operation explanation is added:

$$
\frac{E \oplus\langle x ? t\rangle \vdash e_{1} \sqsupseteq e_{2}}{E \vdash\left(x ? t \ominus e_{1}\right) \sqsupseteq\left(x ? t \ominus e_{2}\right)}
$$

Another extraction rule allows declared parameters to be extracted from within the arguments of an explicit construct:

$$
\begin{aligned}
& \frac{{\operatorname{dec}-\operatorname{pos}_{E}\left(e_{i}, 1\right) \quad x \text {-asm-pos }}_{E}\left(e_{i}, 1\right) \quad 1 \text {-val-dom }{ }_{E}\left(e_{i}, t\right)}{E \vdash \circledast_{e x}\left(e_{1}, \ldots, e_{i}, \ldots, e_{n}\right)} \\
& \sqsupseteq \circledast_{e x}\left(e_{1}, \ldots,\left[x: t \vdash e_{i}(1:=x)\right], \ldots, e_{n}\right)
\end{aligned}
$$

Note, that this rule is reminiscent of the reverse the "eta contraction" principle, i.e.

$$
\left[x: t_{1} \vdash t_{2}(1:=x)\right] \triangleright t_{2},
$$

provided $x$ does not occur free in $t_{2}$. 
Again, in order to allow multiple extractions, the following monotonicity rule for operation explanation is added:

$$
\begin{gathered}
E \oplus\langle x: t\rangle \vdash \circledast_{e x}\left(e_{1}, \ldots, e_{i}, \ldots, e_{n}\right) \sqsupseteq \circledast_{e x}\left(e_{1}^{\prime}, \ldots, e_{i}^{\prime}, \ldots, e_{n}^{\prime}\right) \\
j \neq i \rightarrow E \vdash_{c l} e_{j}^{\prime} \\
\hline E \vdash \circledast_{e x}\left(e_{1}, \ldots,\left[x: t \vdash e_{i}\right], \ldots, e_{n}\right) \\
\sqsupseteq \circledast_{e x}\left(e_{1}^{\prime}, \ldots,\left[x: t \vdash e_{i}^{\prime}\right], \ldots, e_{n}^{\prime}\right)
\end{gathered}
$$

The following rule specifies that explicitation right-commutes with conversion restricted to explicitly valid expressions:

$$
\frac{E \vdash e_{1} \sqsupseteq e_{2} \quad E \vdash e_{2} \sim e_{3} \quad E \vdash_{v a l} e_{2} \quad E \vdash_{\text {val }} e_{3}}{E \vdash e_{1} \sqsupseteq e_{3}}
$$

The next rule is the essential one, because it describes how an extracted implicit definition may be replaced by an appropriate explicit definition:

$$
\frac{E \vdash_{\text {val }} t_{2} E \vdash \operatorname{typ}_{E}\left(t_{2}\right) \dot{\sim} t_{1}}{E \vdash\left[x ? t_{1} \vdash e\right] \sqsupseteq\left[x:=t_{2} \vdash e\right]}
$$

Note the indeterminate nature of this rule, i.e. any valid text $t_{2}$ of type $t_{1}$ is allowed as the instantiation of $x$.

Finally, there is a rule that describes the injection of an expanded argument into a sum with arbitrary (explicitly valid) components:

$$
\begin{gathered}
\forall j \neq i: E \vdash_{v a l} t_{j} \\
\qquad \vdash \circledast_{e x}\left(e_{1}, \ldots, e_{i}, \ldots, e_{n}\right) \\
\sqsupseteq \circledast_{e x}\left(e_{1}, \ldots,\left|x_{1}:={ }^{r} t_{1}\right| \cdots\left|x_{i}:=e_{i}\right| \cdots\left|x_{k}:={ }^{r} t_{k}\right|, \ldots, e_{n}\right)
\end{gathered}
$$

Together with obvious rules for reflexivity and transitivity, this concludes the definition of operation explanation.

Illustrations. A very simple kind of explicitation is the replacement of names by numbers in named operations, e.g. assuming the context from Fig. 1:

$$
\sqsupseteq \underset{\substack{\text { rules.elim } \\ \text { rules.2 } 2}}{\{\mathrm{E} 3 \text {, since elim-com-pos(rules, } 2)\}}
$$

Frequently, such a direct mapping is insufficient to ensure explicit validity. Typically, additional applications need to be introduced as in the following explicitation.

$$
\begin{aligned}
& \text { rules.2(hyp } 2) \\
& \text { ב } \quad\left\{\mathrm{E} 8, \text { where } e_{1}:=\text { rules } 2, e_{2}:=\text { hyp }_{2}, n:=1 \text {, and } t:=\text { prop }\right\} \\
& {\left[p \text { ? prop } \vdash(\text { rules } 2(1:=p))\left(\text { hyp } p_{2}\right)\right]} \\
& \sqsupseteq \quad\left\{\text { EI3, where } t_{1}:=\text { prop and } t_{2}:=b\right\} \\
& {[p:=b \vdash(\text { rules.2(1:=p))(hyp } 2)]} \\
& \sqsupseteq \quad\{\mathrm{E} 12 \text {, based on an obvious conversion }\} \\
& \text { (rules.2(1:=b)) (hyp } 2)
\end{aligned}
$$

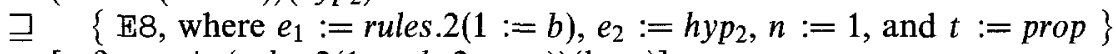

$$
\begin{aligned}
& {[q \text { ? prop } \vdash(\text { rules } 2(1:=b, 2:=q))(\text { hyp } 2)]} \\
& \sqsupseteq \quad\left\{\mathrm{El} 3 \text {, where } t_{1}:=\text { prop and } t_{2}:=c\right\} \\
& {[q:=c \vdash(\text { rules.2(1:=b,2:=q))(hyp })]}
\end{aligned}
$$




$$
\begin{aligned}
& \sqsupseteq\left\{\begin{array}{c}
\text { E12, based on an obvious conversion }\} \\
(\text { rules. } 2(1:=b, 2:=c))(\text { hyp } 2)
\end{array}\right. \\
& \sqsupseteq \quad\{\mathrm{E} 1, \text { since min-dec-pos }(\text { rules } 2(1:=b, 2:=c), 1)\} \\
& (\text { rules. } 2(1:=b, 2:=c))\left(1:=\text { hyp }_{2}\right)
\end{aligned}
$$

To clarify understanding, some instantiations of rule parameters have been given. Note that choosing $b$ and $c$ as arguments of the two new applications is the only possibility to ensure explicit validity. The explicitation of the cut in the Syllogism proof can be illustrated as follows:

$$
\begin{aligned}
& (\text { rules. } 2(1:=a, 1:=c))\left(1:=\text { hyp }_{1}\right) \\
& \$(\text { rules. } 2(1:=b, 1:=c))\left(1:=\text { hyp }_{2}\right) \\
\sqsupseteq & \{\text { E5 }\} \\
& (\text { rules. } 2(1:=a, 1:=c))\left(1:=\text { hyp }_{1}\right) \text { upto } 1 \\
& \$(\text { rules. } 2(1:=b, 1:=c))\left(1:=\text { hyp }_{2}\right) \text { at } 1 \\
\sqsupseteq & \{\text { E10 twice }\} \\
& {\left[u: a \vdash(\text { rules. } 2(1:=a, 1:=c))\left(1:=\text { hyp }_{1}, 1:=u\right)\right] \text { upto } 1 } \\
& \$\left[v: b \vdash(\text { rules } 2(1:=b, 1:=c))\left(1:=\text { hyp }_{2}, 1:=v\right)\right] \text { at } 1
\end{aligned}
$$

Note that explicitation is always concerned with a single operation only. The arguments of that operation must be explicitly valid expressions. The explicitation of the alternative in Fig. 2 runs as follows:

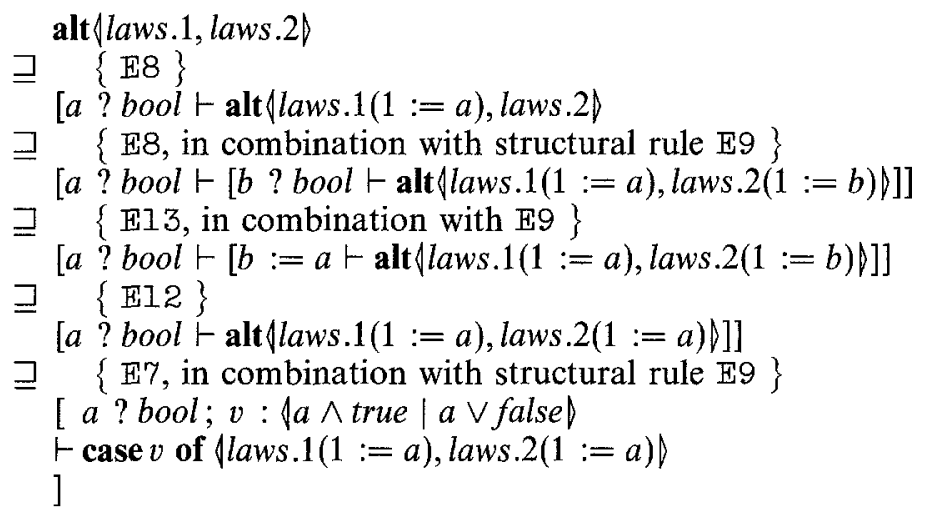

Using the result of this explicitation (corresponding to the definition of simplify in Fig. 2), one can illustrate the explicitation of a single simplification step.

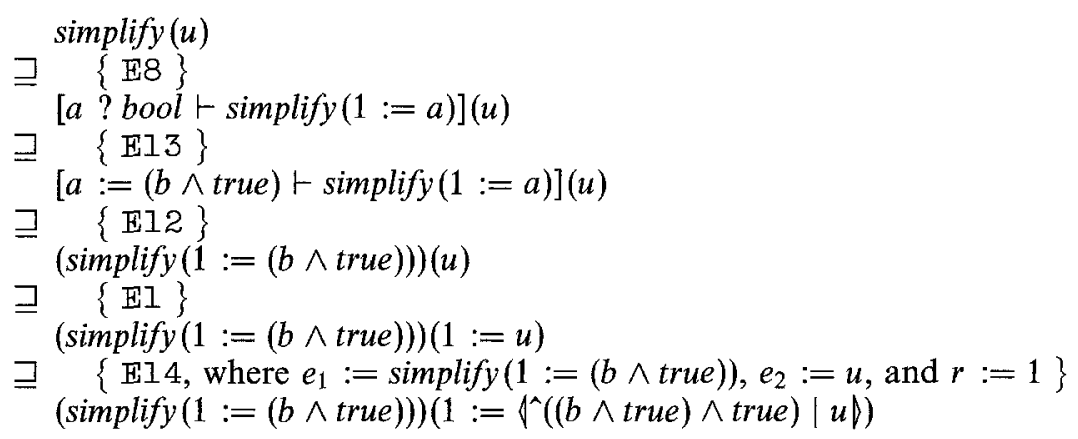

The reduction of the latter expression has been illustrated in section 2.7. 


\subsection{Explanation of Expressions}

In this subsection, explicitation is lifted to explanation, denoted by $E \vdash e_{1} \leadsto e_{2}$. Intuitively, to explain an implicit operation amounts to first explaining its subexpressions and then explicitating the operation on the explained subexpressions into a valid explicit expression. This is made precise by the following rule:

$$
\begin{gathered}
\vdash_{\text {val }} E \quad E \vdash e_{1} \leadsto e_{1}^{\prime} \ldots E \vdash e_{n} \leadsto e_{n}^{\prime} \\
E \vdash \circledast_{i m}\left(e_{1}^{\prime}, \ldots, e_{n}^{\prime}\right) \sqsupseteq e E \vdash_{v a l} e \\
E \vdash \circledast_{i m}\left(e_{1}, \ldots, e_{n}\right) \rightsquigarrow e
\end{gathered}
$$

Note how the validity constraint acts as a filter, i.e. only those explicitations that ensure the explicit validity are allowed. This process is the implicit counterpart to the validity constraints of the explicit part.

The above rule only dealt with implicit operations. Only valid explicit constructs can be used to explain explicit operations, according to the following rule:

$$
\begin{gathered}
\vdash_{\text {val }} E \quad E \vdash e_{1} \rightsquigarrow e_{1}^{\prime} \cdots E \vdash e_{n} \rightsquigarrow e_{n}^{\prime} \\
\operatorname{val}_{E}\left(\circledast_{e x}\left(e_{1}^{\prime}, \ldots, e_{n}^{\prime}\right)\right) \\
E \vdash \circledast_{e x}\left(e_{1}, \ldots, e_{n}\right) \rightsquigarrow \circledast_{e x}\left(e_{1}^{\prime}, \ldots, e_{n}^{\prime}\right)
\end{gathered}
$$

Note that for explicit operations without subexpressions (i.e. $n=0$ ) the above schematic inference rule becomes an axiom. Sequential operations do not have validity constraints and hence they must not be explained. They allow the following monotonicity rule for explanation:

$$
\frac{E \vdash c \leadsto c^{\prime} E \oplus c^{\prime} \vdash e \leadsto e^{\prime}}{E \vdash c \Theta e \leadsto c^{\prime} \Theta e^{\prime}}
$$

The definition of explanation is still incomplete, because iterations have not yet been handled. Iterations can only be explained when occuring in a situation together with certain other constructs. One such situation is $\left(\operatorname{loop} t_{1}\right)\left(t_{2}\right)$, i.e. the text $t_{1}$ is repeatedly applied to $t_{2}$. The explanation of this situation is not very surprising:

$$
\frac{E \vdash t_{1}\left(t_{1}\left(\ldots t_{1}\left(t_{2}\right) \ldots\right)\right) \leadsto t_{3}}{E \vdash\left(\operatorname{loop} t_{1}\right)\left(t_{2}\right) \leadsto t_{3}}
$$

Another situation is $t_{1} \diamond \operatorname{loop} t_{2}$, i.e. the text $t_{2}$ is repeatedly cut with $t_{1}$. The explanation of this situation is as follows:

$$
\frac{E \vdash\left(\cdots\left(\left(t_{1} \otimes t_{2}\right) \otimes t_{2}\right) \cdots \otimes t_{2}\right) \leadsto t_{3}}{E \vdash t_{1} \otimes \operatorname{loop} t_{2} \leadsto t_{3}}
$$

These two rules complete the definition of explanation. It is easy to check that all explanations are explicitly valid, i.e. that $E \vdash e_{1} \leadsto e_{2}$ implies $E \vdash_{v a l} e_{2}$. Figs. 1 and 2 are explanations of Figs. 3 and 4 respectively. Some of the explicitation steps of these explanations have been illustrated in section 3.7.

Remark. Explicitation and explanation are constructive but highly indeterminate operations. A practical approach towards an efficiently automized explicitation process is to guide explicitation by constraints extracted from the expression to be explicitated. Such a guided explicitation process is defined and studied for a kernel subset of Deva in [Web92]. 


\subsection{Implicit Validity}

The notion of implicit validity relative to a valid environment $E$, denoted by $E \vdash_{\text {imval }} e$, is defined as the range of explanation relative to $E$.

$E \vdash_{\text {imval }} e$ if and only if $\vdash_{v a l} E$ and there exists an $e^{\prime}$ with $E \vdash e^{\prime} \rightsquigarrow e$.

\section{Properties of Deva}

This section summarizes basic parts of the language theory of Deva as defined in the previous section. The detailed proofs can be found in [Web90]. There are essentially three "desirable" properties of the language theory:

- Reduction should be confluent, i.e. whenever an expression reduces to two different expressions, then there should be an expression to which these two expressions can be reduced. As a consequence, the result of reduction is independent of the chosen strategy for applying the reduction axioms, e.g. lazy-evaluation, and thus implementations of reduction using any such strategy are guaranteed to be partially correct.

- Explicit validity should be closed with respect to reduction and typing, i.e. the result of reducing or typing an explicitly valid text should still be explicitly valid. This property is quite important for procedures that check explicit validity; it assures that explicit validity need not be rechecked after applying a reduction rule, or after computing a type.

- Finally, reduction should have the strong normalization property on explicitly valid expressions, i.e. all reduction chains starting from an explicitly valid expression should terminate. Quite obviously, this property is essential for the termination of validity checking procedures.

It will turn out that the first two properties can be proven for Deva in full. However, the third property (i.e. strong normalization) can only be proven under a slight restriction (cf. Sec. 4.6) and it remains an open problem whether or not it is satisfied in full.

These three desirable properties are fundamental for many further results. As an illustration of the utilization of these properties two important practical consequences will be briefly presented:

- A decision procedure for explicit validity is outlined.

- It is shown how the problem of proving the adequacy of theory formalizations in Deva can be tackled.

\subsection{Confluence}

The first desirable property, i.e. confluence of reduction, can be stated more precisely as follows:

Theorem 4.1. (Confluence) Consider an arbitrary environment $E$ and an expression $e$ which is closed under $E$. IF there are expressions $e_{1}$ and $e_{2}$ such that $E \vdash e \triangleright e_{1}$ and $E \vdash e \triangleright e_{2}$, then $e_{1}$ and $e_{2}$ have a common reduct, i.e. there exists an expression $e^{\prime}$ such that $E \vdash e_{1} \triangleright e^{\prime}$ and $E \vdash e_{2} \triangleright e^{\prime}$. 
Proof-outline. The basic idea to prove confluence is to partition reduction into a number of subreductions, prove confluence of each of these subreductions, and then prove that their combination preserves the property. The following partition into subreductions is considered:

Definition 4.2. (Partition of $\triangleright$ ) Reduction can be partitioned into the following three subreductions:

a. Let $\triangleright_{\delta}$ denote the restriction of $\triangleright$ to the axioms $R 1$ and $R 2$ for unfolding text and context definitions (cf. Sec. 2.7).

$b$. Let $\nabla_{s}$ denote the restriction of $\triangleright$ to the axioms $R 6$ and $R 20$ to split context joins as left arguments of abstractions and context joins.

c. Let $\triangleright_{r}$ denote the restriction of $\triangleright$ to all axioms except those of of $\triangleright_{\delta}$ and $\triangleright_{s}$.

By a technical, but straightforward analysis, each of these three reductions can be shown to be confluent, i.e. all of them are locally confluent and have the strong normalization property. Furthermore, it can be shown that these reductions commute with each other. For example, if $E \vdash e_{1} \triangleright_{\delta} e_{2}$ and $E \vdash e_{1} \triangleright_{s} e_{3}$ then there exists an $e_{4}$ such that $E \vdash e_{2} \triangleright_{s} e_{4}$ and $E \vdash e_{3} \triangleright_{\delta} e_{4}$. The confluence of reduction follows from the simple fact that confluence is preserved by the union of reductions that commute.

It is interesting to note that in contrast with the well-known confluence proof for $\lambda$-calculus described in e.g. [HS86], the proof in [Web91a] which was outlined above does not introduce the notion of parallel reduction. This necessity arises from the simultaneous substitution of all free occurrences of the formal parameter $x$ by the argument $t$ in the $\lambda$-calculus $\beta$-conversion principle:

$$
(\lambda x . t)\left(t^{\prime}\right) \sim t^{\prime}[x / t]
$$

In contrast, the internalized definition mechanism of Deva allows the individual substitution of free occurrences by $\nabla_{\delta}$ steps. This substitution process is more fine-grained than that of $\lambda$-calculus. As a consequence, confluence can be shown by straightforward decomposition.

Finally, an easy and well-known consequence of confluence is presented.

Corollary 4.3. (Reduction is Church-Rosser) Consider an arbitrary environment $E$ and expressions $e_{1}, e_{2}$ which are closed under $E$. If $E \vdash e_{1} \dot{\sim} e_{2}$ then there exists a common reduct of $e_{1}$ and $e_{2}$.

\subsection{Closure results}

The second desirable property, i.e. the closure results, can be stated more precisely as follows:

Theorem 4.4. (Closure with respect to typing and reduction) Consider an environment $E$ and expressions $e$ and $t$ which are explicitly valid under $E$, i.e. $E \vdash_{\text {val }} e_{1}$ and $E \vdash_{\text {val }} t$. Then the two following properties hold:

- If $E \vdash e_{1} \triangleright e_{2}$ then $E \vdash$ val $e_{2}$, and

- if $\operatorname{typ}_{E}(t)$ is defined then $E \vdash_{\text {val }} \operatorname{typ}_{E}(t)$. 
Proof-outline. Closure with respect to reduction is shown by proving that all reduction axioms preserve closure and that the structural rules of reduction preserve closure. These very technical investigations are based on several substitution lemmas for reduction, typing and validity.

The proof of the second closure result, i.e. closure with respect to typing, essentially involves checking whether or not the validity restrictions of a construct are preserved by typing the construct. It turns out that this can be shown using an additional lemma:

If $E \vdash t_{1} \sim t_{2}$ then $E \vdash \operatorname{typ}_{E}\left(t_{1}\right) \dot{\sim} \operatorname{typ}_{E}\left(t_{2}\right)$ where $t_{1}$ and $t_{2}$ must be valid.

This lemma follows from the first closure result.

\subsection{Strong Normalization}

It is easy to see that reduction does not necessarily terminate on arbitrary closed expressions, for example the expression

$$
[d:=[x: \text { prim } \vdash x(1:=x)] \vdash d(1:=d)]
$$

produces a nonterminating chain of reduction steps. However, the type restrictions imposed upon explicitly valid expressions are designed to exclude such expressions. For example, in the above expression the application $x(1:=x)$ is not explicitly valid because $\operatorname{val}_{E}(x(1:=x))$ implies the existence of a "domain" of $x$ (which is clearly non-existent). In fact, explicitly valid expressions should be strongly normalizable:

Definition 4.5. (Strongly normalizable) An expression e is called strongly normalizable under $E$ if and only if there is no infinite chain of reductions of the following shape:

$$
E \vdash e \triangleright^{1} e_{1} \triangleright^{1} e_{2} \triangleright^{1} \ldots \triangleright^{1} e_{i} \triangleright^{1} \ldots
$$

It turns out that the essential condition that is violated by the above example is the condition that a first domain exists, regardless of whether it conforms to the type of the argument or not. In fact, strong normalization can be shown to hold for a much wider class of expressions, the normable expressions, in which certain generalized structural conditions have to be satisfied. It can be shown that explicitly valid expressions are normable, except for expressions which contain case distinctions. Case distinctions pose a problem because it turns out to be extremely complex to statically, i.e. without performing reduction, figure out which case is eventually selected in a case distinction in a given expression. However, since the overall structure of the expression depends on this selection, the concept of normability could not be defined for expressions containing case distinctions. In the following discussions, the adjective case-free will be used to describe expressions and environments that do not contain case distinctions.

The next step of strong normalization consists of the proof that any normable expression is indeed strongly normalizable. This can be shown with an adaptation of a computability proof presented in [Daa80] for a related system. The idea is to restrict the set of expressions from which reduction terminates, yielding the set of expressions computable under substitution, in such way that it can be shown by simple structural induction that all normable expressions are computable under 
substitution and therefore strongly normalizable. By the previous discussion, this implies the strong normalization of case-free explicitly valid expressions.

Theorem 4.6. (Strong normalization) For any expression e valid under an environment $E$, i.e. $E \vdash_{v a l} e$, it holds that if $e$ and $E$ are case-free then $e$ is strongly normalizable under $E$.

\subsection{Decidability of Case-Free Validity}

Because of the confluence and the strong normalization results, the conversion relation between case-free valid expressions can be decided in a very simple way. To illustrate this point, the following theorem is needed:

Theorem 4.7. (Unique normal form) For any expression e valid under an environment $E$, i.e. $E \vdash_{\text {val }} e$, it holds that if $e$ and $E$ are case-free then any reduction of e terminates and moreover leads to the same expression. This unique result of reduction is denoted by $N F_{E}(e)$ (normal form).

Proof. By strong normalization (cf. Thm. 4.6), the reduction of $e$ indeed terminates and by confluence (cf. Thm. 4.1) it produces a unique result.

To check whether $E \vdash e_{1} \sim e_{2}$ holds for case-free expressions $e_{1}, e_{2}$ that are valid under the case-free environment $E$, it suffices to check whether $N F_{E}\left(e_{1}\right)=$ $N F_{E}\left(e_{2}\right)$. This is correct since by Church-Rosser (cf. Cor. 4.3) there exists an expression $e$ such that both $e_{1}$ and $e_{2}$ reduce to $e$. By closure (cf. Thm. 4.4) $e$ is valid (and obviously case-free). Thus by theorem $4.7, e_{1}, e_{2}$, and $e$ each have unique normal forms which must be obviously identical. This procedure can be extended in a simple (but technical) way to a decision procedure for case-free validity:

Theorem 4.8. (Decidability of case-free explicit validity) For any case-free environment $E$ and expression $e$ it is decidable whether $E \vdash_{\text {val }} e$.

The decidability procedure requires computing the normal forms of the expressions. However, the size of the normal form of an expression may explode exponentially with respect to the size of the original expression, e.g. by complete unfolding of definitions. Thus the procedure is not very practical. There are many ways to develop more efficient procedures to decide conversion, based on the fact that to prove $e_{1} \sim e_{2}$ it suffices to find a (possibly still reducible) common reduct of $e_{1}$ and $e_{2}$. However, the correctness of such a procedure always depends on the properties of confluence and closure (for partial correctness), and strong normalization (for termination).

\subsection{Recursive Characterization of Valid Normal Forms}

Given the existence and uniqueness of normal forms for explicitly valid expressions, it is interesting to look at the structure of these normal forms. The following definition proposes a recursive characterization of valid normal forms. 
Definition 4.9. (Valid normal forms) The set of valid normal forms, denoted by $V N F_{E}$, and the set of "dead ends" i.e. the set of all valid expressions with irreducible elimination constructs at the top, denoted by $D E_{E}$, are defined by mutual recursion as follows:

$$
\begin{aligned}
& V N F_{E}:=\{X \text { (O) } \\
& \cup\left\{\llbracket X \odot e ; c \rrbracket \quad \mid e \in V N F_{E}, c \in V N F_{E \oplus\langle X \odot e)}\right\} \\
& \cup\{\text { prim }\} \\
& \cup\left\{\left[x \odot t_{1} \vdash t_{2}\right] \quad \mid t_{1} \in V N F_{E}, t_{2} \in V N F_{E \oplus\left\langle x \odot t_{1}\right\rangle}\right\} \\
& \cup\left\{\left\langle x_{1}:=t_{1}, \ldots, x_{k}:=t_{k}\right\rangle \mid t_{i} \in V N F_{E} \text { for } i=1, \ldots, k\right\} \\
& \cup\left\{\left|x_{1}:={ }^{r} t_{1}\right| \cdots\left|x_{i}:=t_{i}\right| \cdots\left|x_{k}:={ }^{r} t_{k}\right\rangle\right. \\
& \cup D E_{E} \\
& \left.\mid t_{j} \in V N F_{E} \text { for } j=1, \ldots, k\right\} \\
& \begin{aligned}
D E_{E}:= & \{x \\
& \cup\left\{t_{1}\left(n:=t_{2}\right)\right. \\
& \cup\{t . n \\
& \cup\left\{t_{1} \text { where } n:=t_{2}\right. \\
& \cup\left\{\text { case } t_{1} \text { of } t_{2}\right. \\
& \cup\left\{t_{1} \text { upto } 1 \text { o } t_{2} \text { at } 1\right. \\
& \cup\left\{t_{1} \text { upto } m \text { o> } t_{2} \text { at } 1\right. \\
& \cup\left\{t_{1} \text { upto } m \text { o } t_{2} \text { at } n\right.
\end{aligned}
\end{aligned}
$$

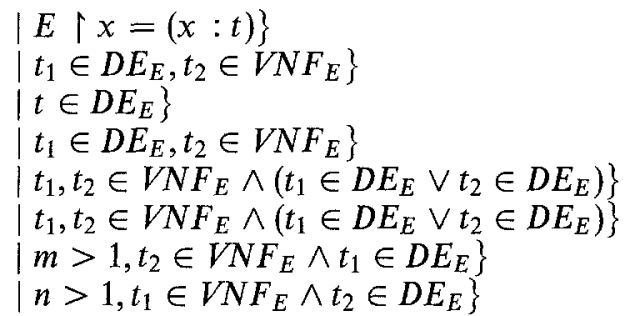

It is rather straightforward to see that an expression explicitly valid under $E$ cannot be reduced any more if and only if it is a member of $V N F_{E}$.

Theorem 4.10. (Valid irreducibility) For any environment $E$ and expressions $e$ with $E \vdash_{v a l} e$ it holds that $e$ is irreducible if and only if $e \in V N F_{E}$.

An application of this result will be presented in the next section.

\subsection{Adequacy of Formalizations}

The goal of this section is to convey some ideas about how to prove adequacy of the formalization of a theory within Deva. Obviously, in order to prove this it is first necessary to define what is meant by a theory. The latter is not obvious. In the context of this presentation the following extremely simplistic definition of a theory will be sufficient.

Definition 4.11. (Theories) $A$ theory $T$ is a pair $\langle$ For, $T h m\rangle$ consisting of a set of formulas, For, and subset of theorems, Thm, of For.

Now assume that a theory $T$ is given and the goal is to adequately formalize that theory in Deva. Before presenting the notion of adequate formalization, one more auxiliary definition is needed.

Definition 4.12. (Inhabitants set) The inhabitants set $I N H_{E}(t)$ of a text $t$ under a case-free environment $E$ is defined as the set of all case-free texts $t_{1}$ such that $E \vdash_{\text {val }} t_{1} \therefore t$.

The basic idea is to formalize the theory $T$ by an environment $E_{T h}$ that consists of an environment $E_{F o r}$ containing declarations producing the formulas of $T$ and 
an environment $E_{A x}$ containing declarations formalizing the axioms and inference rules of $T$. The two systems are related via a bijection.

Definition 4.13. (Adequate formalization) $A$ theory $T=\langle$ For, $T h m\rangle$ is adequately formalized by a case-free environment $E_{T h}=E_{F o r} \oplus E_{A x}$ if and only if $\vdash_{v a l} E_{T h}$ and there is a subset $\mathscr{T}_{\text {For }}$ of $I N H_{E_{F o r}}$ (prim) and a bijection $\alpha:$ For $\rightarrow \mathscr{T}_{\text {For }}$ such that $T h m=\left\{f \in\right.$ For $\left.\mid I N H_{E_{A x}}(\alpha(f)) \neq \emptyset\right\}$.

For convenience, the primitive text prim has been chosen as the type of formulas.

When trying to prove adequacy of formalizations, the difficult point is usually to show that any $f \in$ For with $I N H_{E_{A x}}(\alpha(f)) \neq \emptyset$ is a member of $T h m$. A proof that transforms derivations described by the "inhabitants" of $\alpha(f)$ into derivations of $f$ inside the theory $T$ will face the problem that many such inhabitants, while syntactically completely different, are equivalent modulo conversion. However, as a consequence of confluence and strong normalization the problem can be considerably simplified, because only unique normal forms have to be considered. By using the characterization of valid forms (cf. Sec. 4.10), the following theorem allows the above problem about adequacy proofs to be simplified. It asserts that it suffices to consider those inhabitants which are also valid normal forms.

Theorem 4.14. (Simplification of inhabitance test) For any case-free environment $E$ and text $t$ such that $E \vdash_{v a l} t$ it holds that $I N H_{E}(t) \neq \emptyset$ if and only if $I N H_{E}(t) \cap$ $V N F_{E} \neq \emptyset$.

Proof. In order to show the interesting direction, consider a case-free text $t_{1}$ with $E \vdash_{\text {val }} t_{1} \therefore t$. By strong normalization of case-free validity (cf. Thm. 4.6), $t_{1}$ has a normal form, say $t_{2}$. By closure of explicitly valid expressions with respect to reduction (cf. Thm. 4.4) it follows that $E \vdash_{\text {val }} t_{2}$. By closure of explicit validity with respect to typing and reduction (cf. Thm. 4.4) it follows that also $E \vdash_{\text {val }} t_{2} \therefore t$. By theorem 4.10 it follows that $t_{2}$ must be a member of $V N F_{E}$.

A concrete example of an adequacy proof in a sublanguage of Deva can be found in [Gro90].

\section{Conclusions}

As could be seen, the design choices of the Deva meta-calculus lead to a lean syntax and a somewhat forbidding formal semantics. Yet, the intuitive mastery of the Deva-operations has proved relatively easy for program designers, as illustrated in several detailed case studies. Current research investigates whether this intuitive understanding could be better expressed by an algebraic semantics instead of the operational, $\lambda$-calculus based semantics that underlies the current definition.

Probably the most characteristic language feature of Deva is the syntactical identification of elements and their types, following and extending the ideas of one of the AUTOMATH languages [Ned80]. In addition, Deva offers a number of type structures for the formalization of theories and developments, e.g. named operations, case distinctions, cuts, and contexts. Contexts are lists of declarations and abbreviations together with some simple syntactic operations, i.e. join, application, import, and renaming.

The explanation relation defining the implicit level of Deva was designed to allow the notation and composition of proofs without having to concentrate 
on all the technical overhead resulting from formalization of every detail. When interpreted as a notation for tactics, the implicit level of Deva is much weaker than other tactic notations, as illustrated in e.g. LCF [Pau87], HOL [Gor87], or Mural [JJLM91]. For example, the iteration construct loop is nothing but a flexible shorthand notation for repeated rule applications, in particular it does not introduce the possibility for general recursive definitions. On the other hand, there is no separate meta-notation for tactics in Deva, rather tactical constructs extend the explicit notation and inherit useful features such as the theory system, the abstraction mechanisms etc.

While the explanation of implicit expressions by explicit ones proved sufficient as a descriptive device, its strong nondeterminism precludes any naive implementation of the implicit level in full. Based on the experience gained by ongoing experimentation, current work concentrates on finding ways to guide explanation based on the information available on the implicit level. First technical results can be found in [Web92].

A serious problem arises from the fact that explanation is ambiguous, i.e. an implicit expression may allow more than one explanation. Such ambiguity is harmless when arising from the explanations of implicit proofs since it is irrelevant how the explicit proof looks like, as long as it proves the desired result. However, such ambiguity becomes extremely harmful when arising from the explanation of formulas, for quite obvious reasons. The Deva language itself does not offer any help here, the burden is on the user to discipline himself in order not to be fooled by any harmful ambiguities on the implicit level.

There remain many open points and desirables for a more complete language theory of Deva: For example, the strong normalization result should be established without the restriction to have case-free expressions. Another issue concerns the inclusion of extensional properties, e.g. $\eta$-conversion into the notion of conversion in Deva. Nevertheless, a number of basic positive results have been presented and illustrated to establish a theoretical basis to perform adequacy proofs of Deva-formalizations and to develop sound support systems for Deva.

Up to now there is no model theory for Deva. All consistency proofs have been performed purely on the syntactic definitions. Intuitively, the main obstacle for a naive set-theoretic semantic is the fact that a hierarchy of Deva types is constructed "downwards", i.e. from types to values, as opposed to a hierarchy of typed sets which is constructed "upwards", i.e. from values to types.

\section{Acknowledgements}

This paper is a summary of the theoretical parts of the author's doctoral thesis. He wishes to thank his two supervisors Michel Sintzoff and Stefan Jähnichen for their sustained support. The ideas, concepts, and technical comments of Philippe de Groote have influenced this work to a large degree. Thanks to him and to Martin Simons for his help in numerous discussions on the presentation of the definition of Deva. Many thanks also to Lynn Marshall and to an anonymous referee for their detailed technical and presentational comments.

\section{References}

[Bru72] de Bruijn, N. G.: Lambda calculus notation with nameless dummies, Indagationes Mathematicae, 34:381-392, 1972. 
[Bru80] de Bruijn, N.G.: A Survey of the Project AUTOMATH. In: J.P.Seldin and J.R.Hindley (eds), To H.B.Curry: Essays in Combinatory Logic, Lambda Calculus, and Formalism, pp.589-606, Academic Press, 1980.

[BeS91] Bert, D. and Sebbar, S.: Synthesizing Abstract Data Type Representation in the DEVA Meta-Calculus, Proceedings of the IFIP TC2 Working Conference on Constructing Programs from Specifications, Pacific Grove, Ca., 1991.

[CoH85] Coquand, I. and Huet, G.: Constructions: A Higher Order Proof System for Mechanizing Mathematics. In Proceedings of EUROCAL 85, Linz, Austria, 1985.

[Daa80] van Daalen, D.T.: The Language Theory of AUTOMATH, PhD thesis, Technische Hogeschool Eindhoven, 1980.

[Gab90] Gabriel, R.: (ed.), ESPRIT Project ToolUse, Final Report of the Deva Support Task: Retrospective and Manuals, Arbeitspapiere der GMD, no.425, GMD Karlsruhe, 1990.

[Gab91] Gabriel, R.: Program Transformation Expressed in the DEVA Meta-Calculus, Proceedings of the IFIP TC2 Working Conference on Constructing Programs from Specifications, Pacific Grove, Ca., 1991.

[Gor87] Gordon, M.J.C.: HOL: A Proof Generating System for Higher Order Logic, in G.Birtwhistle and P.A.Subrahmanyam, editors, VLSI pecification, Verification and Synthesis, Kluwer, 1987.

[Gro90] de Groote, Ph.: Définition et Properiéetés d'un métacalcul de répresentation de théories, Thése d'Etat, Unité d'Informatique, Université Catholique de Louvain, Belgium, 1990.

[HHP86] Harper, R., Honsell, F.A. and Plotkin, G.: A Framework for Defining Logics. Proceedings of the 2nd Symposium on Logic in Computer Science, pp. 194-204, IEEE, 1986.

[HiS86] Hindley, J.R. and Seldin, J.P.: Introduction to Combinators and $\lambda$-Calculus, Cambridge University Press, 1986.

[JJLM91] Jones, C.B., Jones, K.D., Lindsay, P.A. and Moore, R.: Mural: A Formal Development Support System. Springer, 1991.

[Laf90] Lafontaine, C.: Formalization of the VDM Reification in the DEVA Meta Calculus. The Human-Leucocyte-Antigen case study. In: M. Broy and C.B. Jones (editors), Programming Concepts and Methods, pp.333-368, North-Holland, 1990.

[Ned80] Nederpelt, R.P.: An Approach to Theorem Proving on the Basis of a Typed Lambda Calculus, LNCS 87, pp.181-190, Springer, 1980.

[Pau87] Paulson, L.; Logic and Computation, Cambridge University Press, 1987

[Sin80] Sintzoff, M.: Understanding and Expressing Software Construction, In P.Pepper, editor, Program Transformations and Programming Environments, pages 169-180, Springer Verlag, 1980

[SWGC89] Sintzoff, M., Weber, M., de Groote, Ph. and Cazin, J.: Definition 1.1 of the Generic Development Language Deva. ToolUse-project, Research report, Unité d'Informatique, Université Catholique de Louvain, Belgium (also available at the author's adress), 1989.

[Web90] Weber, M.: Formalization of the Bird-Meertens Algorithmic Calculus in the Deva MetaCalculus, In: M. Broy and C.B. Jones (editors), Programming Concepts and Methods, pp. 201-232, North-Holland, 1990.

[Web91a] Weber, M.: A Meta-Calculus for Formal System Development, GMD-Bericht Nr. 195, Oldenburg Verlag, München/Wien, 1991.

[Web91b] Weber, M.: Deriving Transitivity of VDM-reification in Deva, Proceedings of the VDM'91 conference, LNCS 551, Springer Verlag, 1991.

[Web92] Weber, M.: Calculating explicit proofs from implicit proofs, internal manuscript, Technical University of Berlin, 1992.

[WSL93] Weber, M., Simons, M. and Lafontaine, C.: The Generic Development Language Deva: Presentation and Case Studies, to be published as an LNCS volume.

Received September 1991

Accepted in revised form September 1992 by C.B. Jones 